\title{
Krasnoselski-Mann-type inertial method for solving split generalized mixed equilibrium and hierarchical fixed point problems
}

Preeyanuch Chuasuk ${ }^{1}$ and Anchalee Kaewcharoen ${ }^{2 *}$

\section{"Correspondence:}

anchaleeka@nu.ac.th

${ }^{2}$ Department of Mathematics

Faculty of Science, Naresuan

University, Phitsanulok, 65000,

Thailand

Full list of author information is

available at the end of the article

\begin{abstract}
In this paper, we present Krasnoselski-Mann-type inertial method for solving split generalized mixed equilibrium and hierarchical fixed point problems for $k$-strictly pseudocontractive nonself-mappings. We establish that the weak convergence of the proposed accelerated iterative method with inertial terms involves a step size which does not require any prior knowledge of the operator norm under several suitable conditions in Hilbert spaces. Finally, the application to a Nash-Cournot oligopolistic market equilibrium model is discussed, and numerical examples are provided to demonstrate the effectiveness of our iterative method.
\end{abstract}

Keywords: Hierarchical fixed point problem; Split generalized mixed equilibrium problem; Maximal monotonicity; Nash-Cournot equilibrium model

\section{Introduction}

Let $H$ be a real Hilbert space with the inner product $\langle\cdot, \cdot\rangle$ and the induced norm $\|\cdot\|$, and let $C$ be a nonempty closed and convex subset of $H$. Let $f: C \rightarrow H$ be a nonlinear mapping, and let $\phi: C \rightarrow \mathbb{R}$ be a function and $F$ be a bifunction from $C \times C$ to $\mathbb{R}$, where $\mathbb{R}$ is the set of real numbers. Then we consider the following generalized mixed equilibrium problem: Find $x^{*} \in C$ such that

$$
F\left(x^{*}, y\right)+\left\langle f x^{*}, y-x^{*}\right\rangle+\phi(y)-\phi\left(x^{*}\right) \geq 0 \quad \text { for all } y \in C .
$$

The set of solutions of (1) is denoted by $\operatorname{GMEP}(F, f, \phi)$.

If $\phi=0$, then the generalized mixed equilibrium problem (1) becomes the following mixed equilibrium problem: Find $x^{*} \in C$ such that

$$
F\left(x^{*}, y\right)+\left\langle f x^{*}, y-x^{*}\right\rangle \geq 0 \quad \text { for all } y \in C .
$$

The set of solutions of (2) is denoted by $\operatorname{MEP}(F, \phi)$.

(c) The Author(s) 2021. This article is licensed under a Creative Commons Attribution 4.0 International License, which permits use, sharing, adaptation, distribution and reproduction in any medium or format, as long as you give appropriate credit to the original author(s) and the source, provide a link to the Creative Commons licence, and indicate if changes were made. The images or other third party material in this article are included in the article's Creative Commons licence, unless indicated otherwise in a credit line to the material. If material is not included in the article's Creative Commons licence and your intended use is not permitted by statutory regulation or exceeds the permitted use, you will need to obtain permission directly from the copyright holder. To view a copy of this licence, visit http://creativecommons.org/licenses/by/4.0/. 
In particular, if $\phi=0$ and $f=0$, then the generalized mixed equilibrium problem (1) becomes the following equilibrium problem: Find $x^{*} \in C$ such that

$$
F\left(x^{*}, y\right) \geq 0 \quad \text { for all } y \in C \text {. }
$$

The set of solutions of (3) is denoted by $\operatorname{EP}(F)$.

On the other hand, if $F\left(x^{*}, y\right)=0, \forall x, y \in C$, then (1) reduces to the following generalized vector variational inequality problem: Find $x^{*} \in C$ such that

$$
\left\langle f x^{*}, y-x^{*}\right\rangle+\phi(y)-\phi\left(x^{*}\right) \geq 0 \quad \text { for all } y \in C .
$$

Problem (4) was discussed in Sun and Chai [45]; it plays a critical role in algorithm design, can be used to measure how much the approximate solution fails to be in the solution set and to analyze the convergence rates of various methods. If we set $F\left(x^{*}, y\right)=0$ and $\phi=0$, $\forall x, y \in C$, then (1) reduces to the classical variational inequality problem (in short, VIP): Find $x^{*} \in C$ such that

$$
\left\langle f x^{*}, y-x^{*}\right\rangle \geq 0 \quad \text { for all } y \in C,
$$

which was first introduced by Giannessi [23]. Recently, several authors have studied and proposed many iterative algorithms for approximating the solutions of variational inequality problem and related optimization problems (see [25, 28, 29]).

The generalized mixed equilibrium problem is very general in the sense that it includes as a special case minimization problems, variational inequality problems, fixed point problems, Nash equilibrium problems in noncooperative games, and many others (see $[2,3,7,12,16,18,21,26,48-50,52])$.

In 1994, Censor and Elfving [14] introduced the following split feasibility problem for modeling inverse problems which arise in phase retrievals and medical image reconstructions. Let $H_{1}$ and $H_{2}$ be two real Hilbert spaces with the inner product $\langle\cdot, \cdot\rangle$ and the norm $\|\cdot\|$. Let $C$ and $Q$ be nonempty closed and convex subsets of $H_{1}$ and $H_{2}$, respectively, and let $A: H_{1} \rightarrow H_{2}$ be a bounded linear operator. The split feasibility problem is formulated as finding a point

$$
x \in C \quad \text { such that } A x \in C .
$$

The split feasibility problem has been applied extensively in many areas of science and engineering such as signal processing, image reconstruction, and intensity modulated radiation therapy. It has received attention of many authors, and various iterative methods have been proposed for finding its solutions (see [13, 15, 17]).

Next, we consider the split generalized mixed equilibrium problem (for short, $\mathrm{S}_{p} \mathrm{GMEP}$ ): Find $x^{*} \in C$ such that

$$
F\left(x^{*}, x\right)+\left\langle f x^{*}, x^{*}-x\right\rangle+\phi(x)-\phi\left(x^{*}\right) \geq 0, \quad \forall x \in C,
$$

and such that

$$
A x^{*} \in Q \text { solves } \quad G\left(A x^{*}, y\right)+\left\langle g\left(A x^{*}\right), A x^{*}-y\right\rangle+\varphi(y)-\varphi\left(A x^{*}\right) \geq 0, \quad \forall y \in Q,
$$


where $F: C \times C \rightarrow \mathbb{R}$ and $G: Q \times Q \rightarrow \mathbb{R}$ are nonlinear bifunctions, $f: C \rightarrow H_{1}$ and $g: Q \rightarrow H_{2}$ are nonlinear mappings, $\phi: C \rightarrow \mathbb{R} \cup\{+\infty\}$ and $\varphi: Q \rightarrow \mathbb{R} \cup\{+\infty\}$ are proper lower semicontinuous and convex functions, and $A: H_{1} \rightarrow H_{2}$ is a bounded linear operator. The set of solutions of the $\mathrm{S}_{p}$ GMEP is denoted by

$$
\Omega=\left\{x^{*} \in \operatorname{GMEP}(F, f, \phi): A x^{*} \in \operatorname{GMEP}(G, g, \varphi)\right\} .
$$

Jolaoso et al. [30] presented the following example to show that $\Omega \neq \emptyset$.

Example 1.1 ([30]) Let $H_{1}=\mathbb{R}^{2}$ with the norm $\|x\|=\sqrt{x_{1}^{2}+x_{2}^{2}}$ for $x=\left(x_{1}, x_{2}\right) \in \mathbb{R}^{2}$, and let $H_{2}=\mathbb{R}$. Let $C=\left\{x=\left(x_{1}, x_{2}\right) \in \mathbb{R}^{2}: x_{2}-x_{1} \geq 1\right\}$ and $Q \subseteq[1, \infty)$. Define $F(x, y)=y_{2}-$ $y_{1}-x_{2}+x_{1}$, where $x=\left(x_{1}, x_{2}\right)$ and $y=\left(y_{1}, y_{2}\right) \in C$. Then $F$ is a bifunction from $C \times C$ to $\mathbb{R}$. Let $f(x)=\phi(x)=x_{2}-x_{1}$, then $\operatorname{GMEP}(F, f, \phi)=\left\{q=\left(q_{1}, q_{2}\right): q_{2}-q_{1}=1\right\}$. Also define $G(u, v)=v-u$ for all $u, v \in Q$, so that $G$ is a bifunction from $Q \times Q$ to $\mathbb{R}$, and let $g(u)=2 u$, $\varphi(u)=u$. For each $x=\left(x_{1}, x_{2}\right) \in H_{1}$, let $A(x)=x_{2}-x_{1}$, so that $A$ is a bounded linear operator from $H_{1}$ to $H_{2}$. Clearly, when $q \in \operatorname{GMEP}(F, f, \phi)$, we have $A q=1 \in \operatorname{GMEP}(G, g, \varphi)$. Thus $\Omega=\{q \in \operatorname{GMEP}(F, f, \phi): A q \in \operatorname{GMEP}(G, g, \varphi)\} \neq \emptyset$.

A nonself-mapping $T: C \rightarrow H_{1}$ is said to be $k$ - strictly pseudocontractive if there exists a constant $k \in[0,1)$ such that

$$
\|T x-T y\|^{2} \leq\|x-y\|^{2}+k\|(I-T) x-(I-T) y\|^{2}, \quad \forall x, y \in H_{1} .
$$

If $k=0$, then $T$ is a nonexpansive nonself-mapping.

It is known that if $\operatorname{Fix}(T)=\left\{x^{*} \in C: T x^{*}=x^{*}\right\} \neq \emptyset$, then $\operatorname{Fix}(T)$ is closed and convex (see [22]).

The hierarchical fixed point problem (in short, HFPP) was introduced by Moudafi and Mainge [39] for a nonexpansive mapping $T$ with respect to another mapping $S$, namely: Find $x^{*} \in \operatorname{Fix}(T)$ such that

$$
\left\langle x^{*}-S x^{*}, x^{*}-x\right\rangle \leq 0 \quad \text { for all } x \in \operatorname{Fix}(T),
$$

where $S: C \rightarrow C$ is a nonexpansive mapping. By using the definition of the normal cone to $\operatorname{Fix}(T)$, i.e.,

$$
N_{\text {Fix }(T)}:= \begin{cases}\left\{u \in H_{1}:\langle y-x, u\rangle \leq 0, \forall y \in \operatorname{Fix}(T)\right\}, & \text { if } x \in \operatorname{Fix}(T), \\ \emptyset, & \text { otherwise }\end{cases}
$$

this amounts to saying that $x^{*} \in \operatorname{Fix}(T)$ satisfies a variational inequality depending on a given criterion $S$, namely: Find $x^{*} \in C$ such that

$$
0 \in(I-S) x^{*}+N_{\mathrm{Fix}(T)} x^{*}
$$

where $I$ is the identity on $C$. It is not hard to check that (8) is equivalent to the fixed point problem: Find $x^{*} \in C$ such that

$$
x^{*} \in P_{\operatorname{Fix}(T)} \cdot S x^{*},
$$


where $P_{\operatorname{Fix}(T)}$ stands for the metric projection on the closed convex set $\operatorname{Fix}(T)$. The solution set of HFPP (8) is denoted by $\Phi=\left\{x^{*} \in C: x^{*}=\left(P_{\mathrm{Fix}(T)} \cdot S\right) x^{*}\right\}$.

At the point, we wish to point out the link with monotone variational inequality on the fixed point set, minimization problems over equilibrium constraints, hierarchical minimization problems, etc.

By setting $S=I-r f$ where $f$ is $\eta$-Lipschitzian and $k$-strongly monotone with $r \in\left(0, \frac{2 k}{\eta^{2}}\right)$, (8) reduces to: Find $x^{*} \in \operatorname{Fix}(T)$ such that

$$
\left\langle x-x^{*}, f x^{*}\right\rangle \geq 0 \quad \text { for all } x \in \operatorname{Fix}(T)
$$

which is a variational inequality in Yamada and Oqura [54]. Now, let $M$ be a maximal monotone operator, by taking $T=J_{\lambda}^{M}=(I-\lambda M)^{-1}$ and $S=I-\gamma \nabla \psi$, where $\psi$ is a convex function such that $\nabla \psi$ is $\eta$-Lipschitzian with $\gamma \in\left(0, \frac{2}{\eta}\right)$ and using the fact that $\operatorname{Fix}\left(J_{\lambda}^{M}\right)=$ $M^{-1}(0)$, then (8) reduces to the following mathematical program with generalized equation constraint:

$$
\min _{0 \in M\left(x^{*}\right)} \psi\left(x^{*}\right)
$$

which was considered by Luo et al. [36]. By taking $M=\partial \varphi$, where $\partial \varphi$ is the subdifferential of a lower semicontinuous convex function, problem (12) is reduced to the following hierarchical minimization problem considered by Cabot [11]:

$$
\min _{x^{*} \in \arg \min \varphi} \psi\left(x^{*}\right)
$$

We note that based upon relation (10), HFPP (8) has the iterative method $x_{n+1}=$ $P_{\mathrm{Fix}(T)}\left(S x_{n}\right)$. It will converge if a fixed point of the operator $P_{\operatorname{Fix}(T)} \cdot S$ exists, and if $S$ is averaged, not just nonexpansive. But calculating $P_{\mathrm{Fix}(T)} \cdot S$ in this case is usually not easy. In 2007, Maudafi [39] introduced the following Krasnoselski-Mann iterative method for solving HFPP (8):

$$
x_{n+1}=\left(1-\alpha_{n}\right) x_{n}+\alpha_{n}\left(\sigma_{n} S x_{n}+\left(1-\sigma_{n} T x_{n}\right)\right) \quad \text { for all } n \geq 0
$$

where $\left\{\alpha_{n}\right\}$ and $\left\{\sigma_{n}\right\}$ are two control sequences in $(0,1)$. The main feature of its corresponding convergence theorems provides a unified frame for analyzing various concrete algorithms (see for instance $[10,55]$ ). It is well known that problem (8) is often used in the area of optimization and related fields, such as signal processing and image reconstruction.

Define a mapping $S_{r_{n}}^{F}: H_{1} \rightarrow C$ and $x \in H_{1}$ as follows:

$$
S_{r_{n}}^{F}(x)=\left\{z \in C: F(z, y)+\frac{1}{r}\langle y-z, z-x\rangle \geq 0, \forall y \in C\right\},
$$

where $F: C \times C \rightarrow \mathbb{R}$ is the bifunction and $r>0$.

In 2017, Kazmi et al. [31] proposed a Krasnoselski-Mann-type iterative method to approximate a common solution set of a hierarchical fixed point problem for nonexpan- 
sive mappings $S, T$ and a split mixed equilibrium problem which was defined as follows:

$$
\begin{aligned}
& x_{0} \in C ; \\
& u_{n}=\left(1-\alpha_{n}\right) x_{n}+\alpha_{n}\left(\beta_{n} S x_{n}+\left(1-\beta_{n}\right) T x_{n}\right) ; \\
& x_{n+1}=S_{r_{n}}^{F}\left(I-r_{n} f\right)\left(u_{n}+\lambda A^{*}\left(S_{r_{n}}^{G}\left(I-r_{n} g\right)-I\right) A u_{n}\right), \quad \forall n \geq 0,
\end{aligned}
$$

where the step size $\lambda \in\left(0, \frac{1}{L}\right), L$ is the spectral radius of the operator $A^{*} A$, and $A^{*}$ is the adjoint of the bounded linear operator $A$. Under some suitable conditions on $\left\{\alpha_{n}\right\},\left\{\beta_{n}\right\}$, and $\left\{r_{n}\right\}$, they proved that the sequence $\left\{x_{n}\right\}$ converges weakly to a solution of hierarchical fixed point and split mixed equilibrium problems.

Recently, Kim and Majee [33] introduced a modified Krasnoselski-Mann iterative method for a common solution of split mixed equilibrium and hierarchical fixed point problems of $k_{i}$-strictly pseudocontractive nonself-mappings $\left\{T_{i}\right\}_{i=1}^{N}$ as follows: For starting point $x_{0} \in C$, define $\left\{x_{n}\right\}$ by

$$
\begin{aligned}
& u_{n}=\left(1-\alpha_{n}\right) x_{n}+\alpha_{n}\left(\beta_{n} S x_{n}+\left(1-\beta_{n}\right) T_{n}^{N} \cdots T_{n}^{1} x_{n}\right) ; \\
& x_{n+1}=S_{r_{n}}^{F}\left(I-r_{n} f\right)\left(u_{n}+\delta_{n} A^{*}\left(S_{r_{n}}^{G}\left(I-r_{n} g\right)-I\right) A u_{n}\right), \quad n \geq 0,
\end{aligned}
$$

where

$$
T_{n}^{i}=\left(1-\gamma_{n}^{i}\right) I+\gamma_{n}^{i} P_{C}\left(\tau_{n}^{i} I+\left(1-\tau_{n}^{i}\right) T^{i}\right), \quad 0 \leq k_{i} \leq \tau_{n}^{i}<1, \gamma_{n}^{i} \in(0,1)
$$

and the step size

$$
\delta_{n}=\frac{\sigma_{n}\left\|\left(S_{r_{n}}^{G}\left(I-r_{n} g\right)-I\right) A x_{n}\right\|}{\left\|A^{*}\left(S_{r_{n}}^{G}\left(I-r_{n} g\right)-I\right) A x_{n}\right\|},
$$

which does not require any prior knowledge of the operator norm. It is well known that the computation or an estimate of the spectral radius of a given operator is very difficult at times. They proved weak convergence to a solution of hierarchical fixed point and split equilibrium problems.

In general, the convergence rate of Krasnoselski-Mann iterative method and hybrid iterative method is slow. In particular, the term $\theta_{n}\left(x_{n}-x_{n-1}\right)$ which is called the inertial extrapolation term was proposed as a remarkable tool for speeding up the convergence properties of iterative methods, and the inertial type algorithm has been studied and modified in various forms by many authors (see [1, 4, 6, 37, 42, 51]). For example, the inertial forward-backward splitting methods [35], the inertial Douglas-Rachford splitting methods [8] and the inertial proximal methods [40], the inertial extragradient methods [27], the inertial subgradient extragradient methods [43], the inertial shrinking projection methods [41].

Motivated and inspired by the work mentioned above, we propose a KrasnoselskiMann-type inertial method for approximating a common solution of a hierarchical fixed point problem for a finite collection of $k$-strictly pseudocontractive nonself-mappings and a split generalized mixed equilibrium problem. Our iterative method combines the 
Krasnoselski-Mann-type iterative method and the inertial term to obtain a new faster iterative method with a step size which does not require any prior knowledge of the operator norm and to prove a weak convergence results under some suitable conditions in real Hilbert spaces. Further, we apply our result to solve a common split mixed equilibrium and hierarchical fixed point problem. Finally, we apply generalized mixed equilibrium problems with Nash-Cournot oligopolistic market equilibrium problems and provide numerical experiments to compare the performances of our proposed iterative method with the method of Kim and Majee [33].

\section{Preliminaries}

In this section, we present some preliminary results that we will use in our results.

Definition 2.1 A mapping $f: H \rightarrow H$ is said to be:

(i) monotone, if

$$
\langle f x-f y, x-y\rangle \geq 0, \quad \forall x, y \in H
$$

(ii) $\alpha$-inverse strongly monotone, if there exists a constant $\alpha>0$ such that

$$
\langle f x-f y, x-y\rangle \geq \alpha\|f x-f y\|^{2}, \quad \forall x, y \in H
$$

(iii) $\beta$-Lipschitz continuous, if there exists a constant $\beta>0$ such that

$$
\|f x-f y\| \leq \beta\|x-y\| \quad \forall x, y \in H .
$$

The normal cone of a nonempty closed convex subset $C$ of $H$ at a point $x \in C$, denoted by $N_{C}(x)$, is defined as

$$
N_{C}(x)=\{u \in H:\langle u, y-x\rangle \leq 0 \text { for all } y \in C\} .
$$

Let $M: H \rightarrow 2^{H}$ be a multivalued operator on $H$. Then the graph $G(M)$ of $M$ is defined by

$$
G(M)=\{(x, y) \in H \times H: y \in M(x)\},
$$

and

(i) the operator $M$ is called a monotone operator if

$$
\langle u-v, x-y\rangle \geq 0, \quad \text { whenever } u \in M(x), v \in M(y) ;
$$

(ii) the operator $M$ is called a maximal monotone operator if $M$ is monotone and the graph of $M$ is not properly contained in the graph of other monotone mappings.

It is clear that a monotone mapping $M$ is maximal if and only if for any $(x, u) \in H \times H$, if $\langle u-v, x-y\rangle \geq 0$ for all $(u, v) \in G(M)$, then $u \in M(x)$ (see [9]).

Lemma 2.2 ([9])

(i) Let $M$ be a maximal monotone mapping on $H$, then the $\left\{t_{n}^{-1} M\right\}$ graph converges to $N_{M^{-1}(0)}$ as $t_{n} \rightarrow 0$ provided that $M^{-1}(0) \neq \emptyset$; 
(ii) Let $\left\{M_{n}\right\}$ be a sequence of maximal monotone mappings on $H$, the graph converges to a mapping $M$ defined on $H$. If $B$ is a Lipschitz maximal monotone mapping on $H$, then the $\left\{B+M_{n}\right\}$ graph converges to $B+M$ and $B+M$ is maximal monotone.

Definition 2.3 A mapping $T: H_{1} \rightarrow H_{1}$ is said to be an averaged mapping if there exists some number $\alpha \in(0,1)$ such that $T=(1-\alpha) I+\alpha S$, where $I: H_{1} \rightarrow H_{1}$ is the identity mapping and $S: H_{1} \rightarrow H_{1}$ is a nonexpansive mapping. An averaged mapping is also nonexpansive and $\operatorname{Fix}(S)=\operatorname{Fix}(T)$.

Lemma 2.4 ([10]) If the mappings $\left\{T_{i}\right\}_{i=1}^{N}$ are averaged and have a common fixed point, then

$$
\bigcap_{i=1}^{N} \operatorname{Fix}\left(T_{i}\right)=\operatorname{Fix}\left(T_{1} T_{2} \cdots T_{N}\right) .
$$

In particular, for $N=2, \operatorname{Fix}\left(T_{1}\right) \cap \operatorname{Fix}\left(T_{2}\right)=\operatorname{Fix}\left(T_{1} T_{2}\right)=\operatorname{Fix}\left(T_{2} T_{1}\right)$.

Lemma 2.5 ([56]) Assume that $S: C \rightarrow H_{1}$ is a $k$-strictly pseudocontractive mapping. Define a mapping $T$ by $T x=\alpha x+(1-\alpha) S x$ for all $x \in H_{1}$, where $\alpha \in[k, 1)$. Then $T$ is a nonexpansive mapping with $\operatorname{Fix}(T)=\operatorname{Fix}(S)$.

Lemma 2.6([56]) Let $T: C \rightarrow H_{1}$ be a $k$-strictly pseudocontractive mapping with $\operatorname{Fix}(T) \neq$ $\emptyset$. Then $\operatorname{Fix}\left(P_{C} T\right)=\operatorname{Fix}(T)$.

Lemma 2.7 ([20]) Let $\left\{\psi_{n}\right\},\left\{\delta_{n}\right\}$, and $\left\{\alpha_{n}\right\}$ be the sequences in $[0,+\infty)$ such that $\psi_{n+1} \leq$ $\psi_{n}+\alpha_{n}\left(\psi_{n}-\psi_{n-1}\right)+\delta_{n}$ for all $n \geq 1, \sum_{n=1}^{\infty} \delta_{n}<+\infty$, and there exists a real number $\alpha$ with $0 \leq \alpha_{n} \leq \alpha<1$ for all $n \geq 1$. Then the following hold:

(i) $\sum_{n \geq 1}\left[\psi_{n}-\psi_{n-1}\right]_{+}<+\infty$, where $[t]_{+}=\max \{t, 0\}$;

(ii) There exists $\psi^{*} \in[0,+\infty)$ such that $\lim _{n \rightarrow+\infty} \psi_{n}=\psi^{*}$.

Lemma 2.8 ([5]) Let $C$ be a nonempty subset of a real Hilbert space $H$ and $\left\{x_{n}\right\}$ be a sequence in $H$ such that the following two conditions hold:

(i) For any $x \in C, \lim _{n \rightarrow \infty}\left\|x_{n}-x\right\|$ exists;

(ii) Every sequential weak cluster point of $\left\{x_{n}\right\}$ is in $C$.

Then $\left\{x_{n}\right\}$ converges weakly to a point in $C$.

\section{Convergence analysis}

In this position, we establish the convergence of the Krasnoselski-Mann-type inertial method and assume that the bifunction $F: C \times C \rightarrow \mathbb{R}$ satisfies the following.

\section{Assumption 3.1}

L1. $F(x, x)=0$ for all $x \in C$;

L2. $F$ is monotone, i.e., $F(x, y)+F(y, x) \leq 0$ for all $x, y \in C$;

L3. For each $x, y, z \in C$, $\limsup _{t \rightarrow 0} F(t z+(1-t) x, y) \leq F(x, y)$;

L4. For each $x \in C, y \rightarrow F(x, y)$ is convex and lower semicontinuous.

The following properties are associated with a nonempty closed and convex subset $C$ of $H$ : 
B1. For each $x \in H$ and $r>0$, there exist a bounded subset $D \subseteq C$ and $y \in C$ such that, for any $z \in D$,

$$
F(z, y)+\phi(y)+\frac{1}{r}\langle y-x, z-x\rangle<\phi(z)
$$

B2. $C$ is a bounded set.

It is easy to show that, under Assumption 3.1, the solution of $\operatorname{GMEP}(F, f, \phi)$ is nonempty, closed, and convex (see, for instance, [44]). We present the following Krasnoselski-Manntype inertial method for solving split generalized mixed equilibrium and hierarchical fixed point problems for $k$-strictly pseudocontractive nonself-mappings.

Algorithm 1 Krasnoselski-Mann-type inertial method

Initialization. Select $\theta \in[0,1)$ and a positive sequence $\left\{\epsilon_{n}\right\} \subset[0, \infty)$ satisfying $\sum_{n=0}^{\infty} \epsilon_{n}<\infty$. Choose $\theta_{n}$ such that $0 \leq \theta_{n} \leq \bar{\theta}_{n}$, where

$$
\bar{\theta}_{n}= \begin{cases}\min \left\{\theta, \frac{\epsilon_{n}}{\left\|x_{n}-x_{n-1}\right\|}\right\}, & \text { if } x_{n} \neq x_{n-1}, \\ \theta, & \text { otherwise }\end{cases}
$$

Choose initial iterates $x_{0}, x_{1} \in C$, and set $n=1$.

Step 1. Compute

$$
\begin{aligned}
& w_{n}=x_{n}+\theta_{n}\left(x_{n}-x_{n-1}\right), \\
& u_{n}=\left(1-\alpha_{n}\right) w_{n}+\alpha_{n}\left(\beta_{n} S w_{n}+\left(1-\beta_{n}\right) T_{n}^{N} \cdots T_{n}^{1} w_{n}\right),
\end{aligned}
$$

where $T_{n}^{i}=\left(1-\gamma_{n}^{i}\right) I+\gamma_{n}^{i} P_{C}\left(\tau_{n}^{i} I+\left(1-\tau_{n}^{i}\right) T^{i}\right), 0 \leq k_{i} \leq \tau_{n}^{i}<1, \gamma_{n}^{i} \in(0,1)$.

Step 2. Compute

$$
x_{n+1}=U\left(u_{n}+\delta_{n} A^{*}(V-I) A u_{n}\right), \quad n \geq 1,
$$

where $U=T_{r_{n}}^{F}\left(I-r_{n} f\right), V=T_{r_{n}}^{G}\left(I-r_{n} g\right)$, and $\delta_{n}=\frac{\sigma_{n}\left\|\left(T_{r_{n}}^{G}\left(I-r_{n} g\right)-I\right) A u_{n}\right\|^{2}}{\left\|A^{*}\left(T_{r_{n}}^{G}\left(I-r_{n} g\right)-I\right) A u_{n}\right\|^{2}}, 0<a \leq \sigma_{n} \leq b<1$.

Set $n=n+1$, and return to Step 1 .

Remark 1 From

$$
\overline{\theta_{n}}=\left\{\begin{array}{ll}
\min \left\{\theta, \frac{\epsilon_{n}}{\left\|x_{n}-x_{n-1}\right\|}\right\}, & \text { if } x_{n} \neq x_{n-1}, \\
\theta, & \text { otherwise, }
\end{array},\right.
$$

we have

$$
\sum_{n=1}^{\infty} \theta_{n}\left(x_{n}-x_{n-1}\right)<\infty
$$


Now, we prove a weak convergence theorem to approximate a common solution of split generalized mixed equilibrium and hierarchical fixed point problems for $k$-strictly pseudocontractive nonself-mappings.

Theorem 3.1 Let $C$ and $Q$ be nonempty closed and convex subsets of real Hilbert spaces $H_{1}$ and $H_{2}$, respectively, and let $A: H_{1} \rightarrow H_{2}$ be a bounded linear operator with its adjoint operator $A^{*}$. Assume that $F: C \times C \rightarrow \mathbb{R}$ and $G: Q \times Q \rightarrow \mathbb{R}$ are bifunctions satisfying Assumption 3.1. Let $f: C \rightarrow H_{1}$ and $g: Q \rightarrow H_{2}$ be $\kappa_{1}, \kappa_{2}$-inverse strongly monotone mappings, $\phi: C \rightarrow \mathbb{R} \cup\{+\infty\}$ and $\varphi: Q \rightarrow \mathbb{R} \cup\{+\infty\}$ be proper lower semicontinuous and convex functions. Let $S: C \rightarrow C$ be a nonexpansive mapping and $\left\{T^{i}\right\}_{i=1}^{N}: C \rightarrow H_{1}$ be $k_{i}$-strictly pseudocontractive nonself-mappings. Assume that either B1 or B2 holds and $\Psi=\Omega \cap \Phi \neq \emptyset$. Let $\left\{x_{n}\right\}$ be the sequence defined by Algorithm 1 and the following conditions are satisfied:

(i) $\liminf _{n \rightarrow \infty} \alpha_{n}>0, \sum_{n=1}^{\infty} \beta_{n}<+\infty$;

(ii) $\lim _{n \rightarrow \infty} \frac{\left\|u_{n}-w_{n}\right\|}{\alpha_{n} \beta_{n}}=0$;

(iii) $\liminf _{n \rightarrow \infty} r_{n}>0$.

Then the sequence $\left\{x_{n}\right\}$ converges weakly to $x^{*} \in \Psi$.

Proof We now divide the remaining proof into several steps.

First, we will prove that $\left\{x_{n}\right\},\left\{u_{n}\right\}$, and $\left\{w_{n}\right\}$ are bounded.

Since $f: C \rightarrow H_{1}$ is a $\kappa_{1}$-inverse strongly monotone mapping, then for any $x, y \in C$, we have

$$
\begin{aligned}
\left\|\left(I-r_{n} f\right) x-\left(I-r_{n} f\right) y\right\|^{2} & =\left\|(x-y)-r_{n}(f x-f y)\right\|^{2} \\
& =\|x-y\|^{2}-r_{n}\left(2 \kappa_{1}-r_{n}\right)\|f x-f y\|^{2}=\|x-y\|^{2},
\end{aligned}
$$

which shows that $\left(I-r_{n} f\right)$ is nonexpansive. Similarly, $\left(I-r_{n} g\right)$ is nonexpansive. So $T_{r_{n}}^{F}(I-$ $\left.r_{n} f\right), T_{r_{n}}^{G}\left(I-r_{n} g\right)$ are nonexpansive. Let $x^{*} \in \Psi$. Then $x^{*} \in \Phi$ and $x^{*} \in \Omega$ which imply that

$$
\begin{aligned}
\left\|w_{n}-x^{*}\right\| & =\left\|x_{n}+\theta_{n}\left(x_{n}-x_{n-1}\right)-x^{*}\right\| \\
& \leq\left\|x_{n}-x^{*}\right\|+\theta_{n}\left\|x_{n}-x_{n-1}\right\| .
\end{aligned}
$$

From Lemma 2.4, Lemma 2.5, and Lemma 2.6, we get $x^{*}=T_{n}^{2} T_{n}^{1} x^{*}$. Hence, we have

$$
\begin{aligned}
\left\|u_{n}-x^{*}\right\|= & \left\|\left(1-\alpha_{n}\right) w_{n}+\alpha_{n}\left(\beta_{n} S w_{n}+\left(1-\beta_{n}\right) T_{n}^{N} \cdots T_{n}^{1} w_{n}\right)-x^{*}\right\| \\
\leq & \left(1-\alpha_{n}\right)\left\|w_{n}-x^{*}\right\|+\alpha_{n}\left[\beta_{n}\left\|S w_{n}-x^{*}\right\|+\left(1-\beta_{n}\right)\left\|T_{n}^{2} T_{n}^{1} w_{n}-x^{*}\right\|\right] \\
\leq & \left(1-\alpha_{n}\right)\left\|w_{n}-x^{*}\right\|+\alpha_{n}\left[\beta_{n}\left\|w_{n}-x^{*}\right\|+\left(1-\beta_{n}\right)\left\|T_{n}^{2} T_{n}^{1} w_{n}-x^{*}\right\|\right. \\
& \left.+\alpha_{n} \beta_{n}\left\|S x^{*}-x^{*}\right\|\right] \\
= & \left\|w_{n}-x^{*}\right\|+\alpha_{n} \beta_{n}\left\|S x^{*}-x^{*}\right\| .
\end{aligned}
$$

Also, since $x^{*} \in \Psi$, we have $U x^{*}=x^{*}$ and $V A x^{*}=A x^{*}$. Let $y_{n}=u_{n}+\delta_{n} A^{*}(V-I) A u_{n}$. Then we get

$$
\begin{aligned}
\left\|y_{n}-x^{*}\right\|^{2} & =\left\|u_{n}+\delta_{n} A^{*}(V-I) A u_{n}-x^{*}\right\|^{2} \\
& \left.=\left\|u_{n}-x^{*}\right\|^{2}+2 \delta_{n}\left\{u_{n}-x^{*}, A^{*}(V-I) A u_{n}\right\rangle+\lambda^{2}\|A\|^{2} \|(V-I) A u_{n}\right) \|^{2} .
\end{aligned}
$$


Observe that

$$
\begin{aligned}
\left\langle u_{n}\right. & \left.-x^{*}, A^{*}(V-I) A u_{n}\right\rangle \\
& =\left\langle A u_{n}-A x^{*},(V-I) A u_{n}\right\rangle \\
& =\left\langle A u_{n}-A x^{*}+(V-I) A u_{n}-(V-I) A u_{n},(V-I) A u_{n}\right\rangle \\
& =\left(\left\langle A u_{n}-A x^{*},(V-I) A u_{n}\right\rangle-\left\|(V-I) A u_{n}\right\|^{2}\right) \\
= & \frac{1}{2}\left(\left\|A u_{n}-A x^{*}\right\|^{2}+\left\|(V-I) A u_{n}\right\|^{2}-\left\|A u_{n}-A x^{*}\right\|^{2}-2\left\|(V-I) A u_{n}\right\|^{2}\right) \\
= & \frac{1}{2}\left(\left\|A u_{n}-A x^{*}\right\|^{2}-\left\|A u_{n}-A x^{*}\right\|^{2}-\left\|(V-I) A u_{n}\right\|^{2}\right) \\
= & -\frac{1}{2}\left\|(V-I) A u_{n}\right\|^{2} .
\end{aligned}
$$

From (16) and (17), we obtain

$$
\left\|y_{n}-x^{*}\right\|^{2} \leq\left\|u_{n}-x^{*}\right\|^{2}-\delta_{n}\left(\left\|(V-I) A u_{n}\right\|^{2}-\delta_{n}\left\|A^{*}(V-I) A u_{n}\right\|^{2}\right) .
$$

It follows that

$$
\begin{aligned}
\left\|x_{n+1}-x^{*}\right\|^{2} & =\left\|U\left(u_{n}+\delta_{n} A^{*}(V-I) A u_{n}\right)-x^{*}\right\|^{2} \\
& =\left\|\left(u_{n}+\delta_{n} A^{*}(V-I) A u_{n}\right)-x^{*}\right\|^{2} \\
& =\left\|y_{n}-x^{*}\right\|^{2} \\
& \leq\left\|u_{n}-x^{*}\right\|^{2}-\delta_{n}\left(\left\|(V-I) A u_{n}\right\|^{2}-\delta_{n}\left\|A^{*}(V-I) A u_{n}\right\|^{2}\right) .
\end{aligned}
$$

From (14),(15), and (19), we get

$$
\begin{aligned}
\left\|x_{n+1}-x^{*}\right\|^{2} & \leq\left\|u_{n}-x^{*}\right\| \\
& \leq\left\|w_{n}-x^{*}\right\|+\alpha_{n} \beta_{n}\left\|S x^{*}-x^{*}\right\| \\
& \leq\left\|x_{n}-x^{*}\right\|+\theta_{n}\left\|x_{n}-x_{n-1}\right\|+\alpha_{n} \beta_{n}\left\|S x^{*}-x^{*}\right\| .
\end{aligned}
$$

Since $\sum_{n=1}^{\infty} \theta_{n}\left\|x_{n}-x_{n-1}\right\|<\infty$ and $\sum_{n=1}^{\infty} \beta_{n}<\infty$, we have $\sum_{n=1}^{\infty} \alpha_{n} \beta_{n}<\infty$. By using Lemma 2.7, we conclude that $\lim _{n \rightarrow \infty}\left\|x_{n}-x^{*}\right\|$ exists. Hence $\left\{x_{n}\right\}$ is bounded, and so $\left\{u_{n}\right\}$ and $\left\{w_{n}\right\}$. Now, from (19), we get

$$
\begin{aligned}
\delta_{n} & \left(\left\|(V-I) A u_{n}\right\|^{2}-\delta_{n}\left\|A^{*}(V-I) A u_{n}\right\|^{2}\right) \\
\leq & \left\|u_{n}-x^{*}\right\|^{2}-\left\|x_{n+1}-x^{*}\right\|^{2} \\
\leq & \left(\left\|w_{n}-x^{*}\right\|+\alpha_{n} \beta_{n}\left\|S x^{*}-x^{*}\right\|\right)^{2}-\left\|x_{n+1}-x^{*}\right\|^{2} \\
\leq & \left(\left\|x_{n}-x^{*}\right\|+\theta_{n}\left\|x_{n}-x_{n-1}\right\|+\alpha_{n} \beta_{n}\left\|S x^{*}-x^{*}\right\|\right)^{2} \\
& \quad-\left\|x_{n+1}-x^{*}\right\|^{2} \\
\leq & \left\|x_{n}-x^{*}\right\|^{2}-\left\|x_{n+1}-x^{*}\right\|^{2}+\alpha_{n} \beta_{n}\left[2\left\|x_{n}-x^{*}\right\|\right.
\end{aligned}
$$




$$
\begin{aligned}
& \left.+\alpha_{n} \beta_{n}\left\|S x^{*}-x^{*}\right\|\right]+2 \theta_{n}\left\|x_{n}-x_{n-1}\right\|\left[\left\|x_{n}-x^{*}\right\|\right. \\
& \left.+\alpha_{n} \beta_{n}\left\|S x^{*}-x^{*}\right\|\right] .
\end{aligned}
$$

Since $\lim _{n \rightarrow \infty} \theta_{n}\left\|x_{n}-x_{n-1}\right\|=0$, we get

$$
\lim _{n \rightarrow \infty} \delta_{n}\left(\left\|(V-I) A u_{n}\right\|^{2}-\delta_{n}\left\|A^{*}(V-I) A u_{n}\right\|^{2}\right)=0,
$$

which by the definition of $\delta_{n}$ implies that

$$
\lim _{n \rightarrow \infty} \frac{\sigma_{n}\left(1-\sigma_{n}\right)\left\|(V-I) A u_{n}\right\|^{4}}{\left\|A^{*}(V-I) A u_{n}\right\|^{2}}=0 .
$$

Since $0<a \leq \sigma_{n} \leq b<1$ and $\left\|(V-I) A u_{n}\right\|$ is bounded, we get

$$
\lim _{n \rightarrow \infty}\left\|(V-I) A u_{n}\right\|=0
$$

Now

$$
\lim _{n \rightarrow \infty}\left\|A^{*}(V-I) A u_{n}\right\|=\lim _{n \rightarrow \infty}\|A\|\left\|(V-I) A u_{n}\right\|=0
$$

So

$$
\lim _{n \rightarrow \infty}\left\|y_{n}-u_{n}\right\|=\lim _{n \rightarrow \infty}\left\|A^{*}(V-I) A u_{n}\right\|=0
$$

Now, we estimate

$$
\begin{aligned}
\left\|x_{n+1}-x_{n}\right\|^{2}= & \left\|\left(x_{n+1}-x^{*}\right)-\left(x_{n}-x^{*}\right)\right\|^{2} \\
\leq & \left\|x_{n+1}-x^{*}\right\|^{2}-\left\|x_{n}-x^{*}\right\|^{2}-2\left\langle x_{n+1}-x_{n}, x_{n}-x^{*}\right\rangle \\
\leq & \left\|x_{n+1}-x^{*}\right\|^{2}-\left\|x_{n}-x^{*}\right\|^{2}-2\left\langle x_{n+1}-p, x_{n}-x^{*}\right\rangle \\
& +2\left\langle x_{n}-p, x_{n}-x^{*}\right\rangle,
\end{aligned}
$$

where $p$ is a weak cluster point of $\left\{x_{n}\right\}$. Since $\lim _{n \rightarrow \infty}\left\|x_{n}-x^{*}\right\|$ exists, then (25) implies that

$$
\lim _{n \rightarrow \infty}\left\|x_{n+1}-x_{n}\right\|=0
$$

From $\sum_{n=1}^{\infty} \theta_{n}\left\|x_{n}-x_{n-1}\right\|<\infty$, we obtain that

$$
\left\|w_{n}-x_{n}\right\|=\left\|x_{n}+\theta_{n}\left(x_{n}-x_{n-1}\right)-x_{n}\right\|=\theta_{n}\left\|x_{n}-x_{n-1}\right\|
$$

and

$$
\lim _{n \rightarrow \infty}\left\|w_{n}-x_{n}\right\|=0
$$


Since $\liminf \operatorname{in}_{n \rightarrow \infty} r_{n}>0$, there is a number $r>0$ such that $r_{n}>r$, we have

$$
\begin{aligned}
\left\|x_{n+1}-x^{*}\right\|^{2} & =\left\|T_{r_{n}}^{F}(I-r f) y_{n}-T_{r_{n}}^{F}(I-r f) x^{*}\right\|^{2} \\
& =\left\|(I-r f) y_{n}-(I-r f) x^{*}\right\|^{2} \\
& =\left\|y_{n}-x^{*}\right\|^{2}-2 r\left(y_{n}-x^{*}, f y_{n}-f x^{*}\right)+r^{2}\left\|f y_{n}-f x^{*}\right\|^{2} \\
& \leq\left\|y_{n}-x^{*}\right\|^{2}-2 r \kappa_{1}\left\|f y_{n}-f x^{*}\right\|^{2}+r^{2}\left\|f y_{n}-f x^{*}\right\|^{2} \\
& =\left\|y_{n}-x^{*}\right\|^{2}-r\left(2 \kappa_{1}-r\right)\left\|f y_{n}-f x^{*}\right\|^{2} \\
& \leq\left\|y_{n}-x^{*}\right\|^{2}-r\left(2 \kappa_{1}-r\right)\left\|f y_{n}-f x^{*}\right\|^{2} .
\end{aligned}
$$

Thus

$$
\begin{aligned}
r\left(2 \kappa_{1}-r\right)\left\|f y_{n}-f x^{*}\right\|^{2} \leq & \left\|y_{n}-x^{*}\right\|^{2}-\left\|x_{n+1}-x^{*}\right\|^{2} \\
\leq & \left\|u_{n}-x^{*}\right\|^{2}-\left\|x_{n+1}-x^{*}\right\|^{2} \\
\leq & \left(\left\|w_{n}-x^{*}\right\|+\alpha_{n} \beta_{n}\left\|S x^{*}-x^{*}\right\|\right)^{2}-\left\|x_{n+1}-x^{*}\right\|^{2} \\
\leq & \left(\left\|x_{n}-x^{*}\right\|+\theta_{n}\left\|x_{n}-x_{n-1}\right\|+\alpha_{n} \beta_{n}\left\|S x^{*}-x^{*}\right\|\right)^{2} \\
& \quad-\left\|x_{n+1}-x^{*}\right\|^{2} \\
\leq & \left\|x_{n}-x^{*}\right\|^{2}-\left\|x_{n+1}-x^{*}\right\|^{2}+\alpha_{n} \beta_{n}\left[2\left\|x_{n}-x^{*}\right\|\right. \\
& \left.+\alpha_{n} \beta_{n}\left\|S x^{*}-x^{*}\right\|\right]+2 \theta_{n}\left\|x_{n}-x_{n-1}\right\|\left[\left\|x_{n}-x^{*}\right\|\right. \\
& \left.+\alpha_{n} \beta_{n}\left\|S x^{*}-x^{*}\right\|\right] .
\end{aligned}
$$

Since $r\left(2 \kappa_{1}-r\right)>0, \lim _{n \rightarrow \infty} \theta_{n}\left\|x_{n}-x_{n-1}\right\|=0, \sum_{n=1}^{\infty} \beta_{n}<+\infty$, and $\lim _{n \rightarrow \infty}\left\|x_{n}-x^{*}\right\|$ exists, we have

$$
\lim _{n \rightarrow \infty}\left\|f y_{n}-f x^{*}\right\|=0
$$

Since $T_{r_{n}}^{F}$ is firmly nonexpansive, we get

$$
\begin{aligned}
\left\|x_{n+1}-x^{*}\right\|^{2}= & \left\|T_{r_{n}}^{F}\left(I-r_{n} f\right) y_{n}-T_{r_{n}}^{F}\left(I-r_{n} f\right) x^{*}\right\|^{2} \\
\leq & \left\langle(I-r f) y_{n}-(I-r f) x^{*}, x_{n+1}-x^{*}\right\rangle \\
= & \frac{1}{2}\left[\left\|(I-r f) y_{n}-(I-r f) x^{*}\right\|^{2}+\left\|x_{n+1}-x^{*}\right\|^{2}\right. \\
& \left.-\left\|y_{n}-x_{n+1}-r\left(f y_{n}-f x^{*}\right)\right\|^{2}\right] \\
\leq & \frac{1}{2}\left[\left\|y_{n}-x^{*}\right\|^{2}+\left\|x_{n+1}-x^{*}\right\|^{2}\right. \\
& -\left\|y_{n}-x_{n+1}\right\|^{2}+2 r\left(y_{n}-x_{n+1}, f y_{n}-f x^{*}\right\rangle \\
& \left.-r^{2}\left\|f y_{n}-f x^{*}\right\|^{2}\right] \\
\leq & \frac{1}{2}\left[\left\|y_{n}-x^{*}\right\|^{2}+\left\|x_{n+1}-x^{*}\right\|^{2}\right. \\
& \left.-\left\|y_{n}-x_{n+1}\right\|^{2}+2 r\left\|y_{n}-x_{n+1}\right\|\left\|f y_{n}-f x^{*}\right\|\right]
\end{aligned}
$$


which in turn yields

$$
\left\|x_{n+1}-x^{*}\right\|^{2} \leq\left\|y_{n}-x^{*}\right\|^{2}-\left\|y_{n}-x_{n+1}\right\|^{2}+2 r\left\|y_{n}-x_{n+1}\right\|\left\|f y_{n}-f x^{*}\right\|,
$$

and this together with (14), (15), (16), and (18) implies that

$$
\begin{aligned}
\left\|y_{n}-x_{n+1}\right\|^{2} \leq & \left\|y_{n}-x^{*}\right\|^{2}-\left\|x_{n+1}-x^{*}\right\|^{2}+2 r\left\|y_{n}-x_{n+1}\right\|\left\|f y_{n}-f x^{*}\right\| \\
\leq & \left\|u_{n}-x^{*}\right\|^{2}-\left\|x_{n+1}-x^{*}\right\|^{2}+2 r\left\|y_{n}-x_{n+1}\right\|\left\|f y_{n}-f x^{*}\right\| \\
\leq & \left(\left\|w_{n}-x^{*}\right\|+\alpha_{n} \beta_{n}\left\|S x^{*}-x^{*}\right\|\right)^{2}-\left\|x_{n+1}-x^{*}\right\|^{2} \\
& \quad+2 r\left\|y_{n}-x_{n+1}\right\|\left\|f y_{n}-f x^{*}\right\| \\
\leq & \left(\left\|x_{n}-x^{*}\right\|+\theta_{n}\left\|x_{n}-x_{n-1}\right\|+\alpha_{n} \beta_{n}\left\|S x^{*}-x^{*}\right\|\right)^{2}-\left\|x_{n+1}-x^{*}\right\|^{2} \\
& +2 r\left\|y_{n}-x_{n+1}\right\|\left\|f y_{n}-f x^{*}\right\| \\
\leq & \left\|x_{n}-x^{*}\right\|^{2}-\left\|x_{n+1}-x^{*}\right\|^{2}+\alpha_{n} \beta_{n}\left[2\left\|x_{n}-x^{*}\right\|\right. \\
& \left.+\alpha_{n} \beta_{n}\left\|S x^{*}-x^{*}\right\|\right]+2 \theta_{n}\left\|x_{n}-x_{n-1}\right\|\left[\left\|x_{n}-x^{*}\right\|\right. \\
& \left.+\alpha_{n} \beta_{n}\left\|S x^{*}-x^{*}\right\|\right]+2 r\left\|y_{n}-x_{n+1}\right\|\left\|f y_{n}-f x^{*}\right\| .
\end{aligned}
$$

From (28), we get

$$
\lim _{n \rightarrow \infty}\left\|y_{n}-x_{n+1}\right\|=0
$$

It follows that

$$
\left\|x_{n}-y_{n}\right\| \leq\left\|x_{n}-x_{n+1}\right\|+\left\|x_{n+1}-y_{n}\right\|
$$

Thus

$$
\lim _{n \rightarrow \infty}\left\|y_{n}-x_{n}\right\|=0
$$

Since

$$
\left\|x_{n}-u_{n}\right\| \leq\left\|x_{n}-y_{n}\right\|+\left\|y_{n}-u_{n}\right\|,
$$

from (24) and (30), we have

$$
\lim _{n \rightarrow \infty}\left\|x_{n}-u_{n}\right\|=0 .
$$

Furthermore,

$$
\left\|u_{n}-w_{n}\right\| \leq\left\|u_{n}-x_{n}\right\|+\left\|x_{n}-w_{n}\right\|,
$$

from (24) and (27), we have

$$
\lim _{n \rightarrow \infty}\left\|u_{n}-w_{n}\right\|=0
$$


Next, we will show that $x^{*} \in \Psi$. Since $T_{n}^{2} T_{n}^{1}$ is an averaged mapping, it is nonexpansive. Using the boundedness of $\left\{w_{n}\right\}$ and the nonexpansivity of $S$, there exists $K>0$ such that $\left\|S w_{n}-T_{n}^{2} T_{n}^{1} w_{n}\right\| \leq K$ for all $n \geq 1$. Consider

$$
\begin{aligned}
\left\|u_{n}-T_{n}^{2} T_{n}^{1} w_{n}\right\|= & \left\|\left(1-\alpha_{n}\right) w_{n}+\alpha_{n}\left(\beta_{n} S w_{n}+\left(1-\beta_{n}\right) T_{n}^{2} T_{n}^{1} w_{n}\right)-T_{n}^{2} T_{n}^{1} w_{n}\right\| \\
\leq & \left(1-\alpha_{n}\right)\left\|w_{n}-T_{n}^{2} T_{n}^{1} w_{n}\right\|+\alpha_{n} \beta_{n}\left\|S w_{n}-T_{n}^{2} T_{n}^{1} w_{n}\right\| \\
\leq & \left(1-\alpha_{n}\right)\left\|u_{n}-w_{n}\right\|+\left(1-\alpha_{n}\right)\left\|u_{n}-T_{n}^{2} T_{n}^{1} w_{n}\right\| \\
& +\alpha_{n} \beta_{n}\left\|S w_{n}-T_{n}^{2} T_{n}^{1} w_{n}\right\|,
\end{aligned}
$$

which implies that

$$
\begin{aligned}
\alpha_{n}\left\|u_{n}-T_{n}^{2} T_{n}^{1} w_{n}\right\| & \leq\left(1-\alpha_{n}\right)\left\|u_{n}-w_{n}\right\|+\alpha_{n} \beta_{n}\left\|S w_{n}-T_{n}^{2} T_{n}^{1} w_{n}\right\| . \\
& \leq\left(1-\alpha_{n}\right)\left\|u_{n}-w_{n}\right\|+\alpha_{n} \beta_{n} K .
\end{aligned}
$$

So

$$
\left\|u_{n}-T_{n}^{2} T_{n}^{1} w_{n}\right\| \leq \frac{\left\|u_{n}-w_{n}\right\|}{\alpha_{n}}+\beta_{n} K .
$$

It follows from conditions (I)-(II) that

$$
\lim _{n \rightarrow \infty}\left\|u_{n}-T_{n}^{2} T_{n}^{1} w_{n}\right\|=0
$$

Since

$$
\left\|w_{n}-T_{n}^{2} T_{n}^{1} w_{n}\right\| \leq\left\|w_{n}-u_{n}\right\|+\left\|u_{n}-T_{n}^{2} T_{n}^{1} w_{n}\right\|,
$$

from (32) and (34), we get

$$
\lim _{n \rightarrow \infty}\left\|w_{n}-T_{n}^{2} T_{n}^{1} w_{n}\right\|=0
$$

Since $\left\{w_{n}\right\}$ is bounded, there exists a subsequence $\left\{w_{n_{k}}\right\}$ that weakly converges to $x^{*}$. Using the boundedness of $\left\{\gamma_{n}^{i}\right\}$ for $i=1,2$, we can assume that $\gamma_{n_{k}}^{i} \rightarrow \gamma_{\infty}^{i}$ as $k \rightarrow \infty$, where $0<$ $\gamma_{\infty}^{i}<1$ for $i=1,2$. Let

$$
T_{\infty}^{i}=\left(1-\gamma_{\infty}^{i}\right) I+\gamma_{\infty}^{i} P_{C}\left(\tau^{i} I+\left(1-\tau^{i}\right) T^{i}\right), \quad \forall i=1,2
$$

Now, by Lemma 2.5 and Lemma 2.6, $\operatorname{Fix}\left(P_{C}\left(\tau^{i} I+\left(1-\tau^{i}\right) T^{i}\right)\right)=\operatorname{Fix}\left(T^{i}\right)$. Again, since $P_{C}\left(\tau^{i} I+\left(1-\tau^{i}\right) T^{i}\right)$ is a nonexpansive mapping, $T_{\infty}^{i}$ is averaged and $\operatorname{Fix}\left(T_{\infty}^{i}\right)=\operatorname{Fix}\left(T^{i}\right)$ for $i=1,2$. Since

$$
\operatorname{Fix}\left(T_{\infty}^{1}\right) \cap \operatorname{Fix}\left(T_{\infty}^{2}\right)=\operatorname{Fix}\left(T^{1}\right) \cap \operatorname{Fix}\left(T^{2}\right)=\operatorname{Fix}(\Psi) \neq \emptyset
$$

by Lemma 2.4, we get

$$
\operatorname{Fix}\left(T_{\infty}^{1} T_{\infty}^{2}\right)=\operatorname{Fix}\left(T_{\infty}^{1}\right) \cap \operatorname{Fix}\left(T_{\infty}^{2}\right)=\operatorname{Fix}(\Psi)
$$


Since

$$
\left\|T_{n_{k}}^{i}(t)-T_{\infty}^{i}(t)\right\| \leq\left|\gamma_{n_{k}}^{i}-\gamma_{\infty}^{i}\right|\left(\|t\|+\left\|P_{C}\left(\tau^{i} t+\left(1-\tau^{i}\right) T^{i}(t)\right)\right\|\right),
$$

we get

$$
\lim _{k \rightarrow \infty} \sup _{t \in B}\left\|T_{n_{k}}^{i}(t)-T_{\infty}^{i}(t)\right\|=0
$$

where $B$ is an arbitrary bounded subset of $H_{1}$. Also, we have

$$
\begin{aligned}
\left\|w_{n_{k}}-T_{\infty}^{2} T_{\infty}^{1} w_{n_{k}}\right\| \leq & \left\|w_{n_{k}}-T_{n_{k}}^{2} T_{n_{k}}^{1} w_{n_{k}}\right\|+\left\|T_{n_{k}}^{2} T_{n_{k}}^{1} w_{n_{k}}-T_{\infty}^{2} T_{n_{k}}^{1} w_{n_{k}}\right\| \\
& +\left\|T_{\infty}^{2} T_{n_{k}}^{1} w_{n_{k}}-T_{\infty}^{2} T_{\infty}^{1} w_{n_{k}}\right\| \\
\leq & \left\|w_{n_{k}}-T_{n_{k}}^{2} T_{n_{k}}^{1} w_{n_{k}}\right\|+\left\|T_{n_{k}}^{2} T_{n_{k}}^{1} w_{n_{k}}-T_{\infty}^{2} T_{n_{k}}^{1} w_{n_{k}}\right\| \\
& +\left\|T_{n_{k}}^{1} w_{n_{k}}-T_{\infty}^{1} w_{n_{k}}\right\| \\
\leq & \left\|w_{n_{k}}-T_{n_{k}}^{2} T_{n_{k}}^{1} w_{n_{k}}\right\|+\sup _{t \in B_{1}}\left\|T_{n_{k}}^{2} t-T_{\infty}^{2} t\right\| \\
& +\sup _{t \in B_{2}}\left\|T_{n_{k}}^{1} t-T_{\infty}^{1} t\right\|,
\end{aligned}
$$

where $B_{1}$ is a bounded subset of $\left\{T_{n_{k}}^{1} x_{n_{k}}\right\}$ and $B_{2}$ is a bounded subset of $\left\{x_{n_{k}}\right\}$. It follows from (35), (36), and (37) that

$$
\lim _{k \rightarrow \infty}\left\|w_{n_{k}}-T_{\infty}^{2} T_{\infty}^{1} w_{n_{k}}\right\|=0
$$

Hence, from Lemma 2.4, we get $x^{*} \in \operatorname{Fix}\left(T_{\infty}^{2} T_{\infty}^{1}\right)=\operatorname{Fix}\left(T^{1}\right) \cap \operatorname{Fix}\left(T^{2}\right)$. Again from Algorithm 1 , we have

$$
u_{n}-w_{n}=\alpha_{n}\left(\beta_{n}\left(S w_{n}-w_{n}\right)-\left(1-\beta_{n}\right)\left(T_{n}^{2} T_{n}^{1} w_{n}-w_{n}\right)\right)
$$

and hence

$$
\frac{1}{\alpha_{n} \beta_{n}}\left(w_{n}-u_{n}\right)=\left((I-S)+\left(\frac{1-\beta_{n}}{\beta_{n}}\right)\left(I-T_{n}^{2} T_{n}^{1}\right)\right) w_{n}
$$

By Lemma 2.2(i) guarantees that the operator sequence $\left\{\left(\frac{1-\beta_{n}}{\beta_{n}}\right)\left(I-T_{n}^{2} T_{n}^{1}\right)\right\}$ graph converges to $N_{\mathrm{Fix}\left(T^{1}\right) \cap \mathrm{Fix}\left(T^{2}\right)}$, and hence it follows from Lemma 2.2(ii) that the operator sequence $\{(I-$ $\left.S)+\left(\frac{1-\beta_{n}}{\beta_{n}}\right)\left(I-T_{n}^{2} T_{n}^{1}\right)\right\}$ graph converges to $(I-S)+N_{\operatorname{Fix}\left(T^{1}\right) \cap \operatorname{Fix}\left(T^{2}\right)}$. Now, by replacing $n$ with $n_{k}$ and by passing to the limit in (38) as $k \rightarrow \infty$ and by taking into account the fact that $\lim _{n \rightarrow \infty} \frac{1}{\alpha_{n} \beta_{n}}\left\|w_{n}-u_{n}\right\|=0$ and that the graph of $(I-S)+N_{\operatorname{Fix}\left(T^{1}\right) \cap \operatorname{Fix}\left(T^{2}\right)}$ is weakly-strongly closed, we obtain that $0 \in(I-S) x^{*}+N_{\mathrm{Fix}\left(T^{1}\right) \cap \mathrm{Fix}\left(T^{2}\right)} x^{*}$, so $x^{*} \in \Phi$.

Finally, we show that $x^{*} \in \Omega$. Since $x_{n+1}=U\left(y_{n}\right)=T_{r_{n}}^{F}\left(I-r_{n} f\right)\left(y_{n}\right)$, we have

$$
F\left(x_{n+1}, y\right)+\left\langle f y_{n}, y-x_{n+1}\right\rangle+\phi(y)-\phi\left(x_{n+1}\right)+\frac{1}{r_{n}}\left\langle y-x_{n+1}, x_{n+1}-y_{n}\right\rangle \geq 0, \quad \forall y \in C .
$$


Since $F$ is monotone, the above inequality implies

$$
\left\langle f y_{n}, y-x_{n+1}\right\rangle+\phi(y)-\phi\left(x_{n+1}\right)+\frac{1}{r_{n}}\left\langle y-x_{n+1}, x_{n+1}-y_{n}\right\rangle \geq F\left(y, x_{n+1}\right), \quad \forall y \in C,
$$

and hence replacing $n$ with $n_{k}$ in the above inequality, we have

$$
\begin{aligned}
& \left\langle f y_{n_{k}}, y-x_{n_{k}+1}\right\rangle+\left\langle y-x_{n_{k}+1}, \frac{x_{n_{k}+1}-y_{n_{k}}}{r_{n_{k}}}\right\rangle \\
& \geq F\left(y, x_{n_{k}+1}\right)-\phi(y)+\phi\left(x_{n_{k}}+1\right), \quad \forall y \in C .
\end{aligned}
$$

Further, for any $t \in(0,1]$ and $y \in C$, let $y_{t}=t y+(1-t) x^{*}$. Since $x^{*} \in C$ and $y \in C$, then $y_{t} \in C$. From the monotonicity of $F$, the above inequality implies

$$
\begin{aligned}
\left\langle y_{t}-x_{n_{k}+1}, f y_{t}\right\rangle \geq & \left\langle y_{t}-x_{n_{k}+1}, f y_{t}-f x_{n_{k}+1}\right\rangle+\left\langle y_{t}-x_{n_{k}+1}, f x_{n_{k}+1}-f y_{n_{k}}\right\rangle \\
& -\left\langle y_{t}-x_{n_{k}+1}, \frac{x_{n_{k}+1}-y_{n_{k}}}{r_{n_{k}}}\right\rangle+F\left(y_{t}, x_{n_{k}+1}\right)-\phi\left(y_{t}\right)+\phi\left(x_{n_{k}+1}\right) .
\end{aligned}
$$

Since the sequences $\left\{x_{n}\right\},\left\{y_{n}\right\}$, and $\left\{w_{n}\right\}$ have the same asymptotic behavior and $x_{n_{k}} \rightarrow x^{*}$, there exists a subsequence $\left\{y_{n_{k}}\right\}$ of $\left\{y_{n}\right\}$ such that $y_{n_{k}} \rightarrow x^{*}$. Since $\lim _{k \rightarrow \infty}\left\|x_{n_{k}+1}-y_{n_{k}}\right\|=0$ and $f$ is Lipschitz continuous, we have $\lim _{k \rightarrow \infty}\left\|f x_{n_{k}+1}-f y_{n_{k}}\right\|=0$. From the condition of $\liminf _{n \rightarrow \infty} r_{n}>0$, there exists a number $r>0$ such that $\liminf _{n \rightarrow \infty} r_{n}=r$, which implies that

$$
\begin{aligned}
\lim _{k \rightarrow \infty} \frac{\left\|x_{n_{k}+1}-y_{n_{k}}\right\|}{r_{n_{k}}} & \leq \frac{\lim _{k \rightarrow \infty}\left\|x_{n_{k}+1}-y_{n_{k}}\right\|}{\lim _{k \rightarrow \infty} r_{n_{k}}} \\
& =\frac{1}{r} \lim _{k \rightarrow \infty}\left\|x_{n_{k}+1}-y_{n_{k}}\right\|=0 .
\end{aligned}
$$

From the monotonicity of $f$, we have

$$
\left\langle y_{t}-x_{n_{k}+1}, f y_{t}-f x_{n_{k}+1}\right\rangle \geq 0
$$

Therefore, by L4 and the weak lower semicontinuity of $\phi$, taking the limit of (40) as $k \rightarrow \infty$, we get

$$
\left\langle y_{t}-x^{*}, f y_{t}\right\rangle \geq F\left(y_{t}, x^{*}\right)+\phi\left(x^{*}\right)-\phi\left(y_{t}\right)
$$

The convexity of $F$ and (41) imply that

$$
\begin{aligned}
0 & =F\left(y_{t}, y_{t}\right)+\phi\left(y_{t}\right)-\phi\left(y_{t}\right) \\
& \leq t F\left(y_{t}, y\right)+(1-t) F\left(y_{t}, x^{*}\right)+t F(y)+(1-t) \phi\left(x^{*}\right)-\phi\left(y_{t}\right) \\
& =t\left(F\left(y_{t}, y\right)+\phi(y)-\phi\left(y_{t}\right)\right)+(1-t)\left(F\left(y_{t}, x^{*}\right)+\phi\left(x^{*}\right)-\phi\left(y_{t}\right)\right) \\
& \leq t\left(F\left(y_{t}, y\right)+\phi(y)-\phi\left(y_{t}\right)\right)+(1-t)\left\langle y_{t}-x^{*}, f y_{t}\right\rangle \\
& \leq t\left(F\left(y_{t}, y\right)+\phi(y)-\phi\left(y_{t}\right)\right)+(1-t) t\left(y-x^{*}, f y_{t}\right\rangle,
\end{aligned}
$$




$$
F\left(y_{t}, y\right)+\phi(y)-\phi\left(y_{t}\right)+(1-t)\left\langle y-x^{*}, f y_{t}\right\rangle \geq 0
$$

Letting $t \rightarrow 0$, then for each $y \in C$, we have

$$
F\left(x^{*}, y\right)+\phi(y)-\phi\left(x^{*}\right)+\left\langle y-x^{*}, f x^{*}\right\rangle \geq 0 .
$$

This implies that $x^{*} \in \operatorname{GMEP}(F, f, \phi)$. Since $A$ is a bounded linear operator, then $A u_{n_{k}} \rightarrow$ $A x^{*}$. Now, setting $s_{n_{k}}=A u_{n_{k}}-V A u_{n_{k}}$, it follows from (22) that $\lim _{k \rightarrow \infty} s_{n_{k}}=0$ and $A u_{n_{k}}-$ $s_{n_{k}}=V A u_{n_{k}}$. Therefore,

$$
\begin{gathered}
G\left(A u_{n_{k}}-s_{n_{k}}, z\right)+\left\langle g A u_{n_{k}}, z-\left(A u_{n_{k}}-s_{n_{k}}\right)\right\rangle+\varphi(z)-\varphi\left(A u_{n_{k}}-s_{n_{k}}\right) \\
+\frac{1}{r_{n}}\left\langle z-\left(A u_{n_{k}}-s_{n_{k}}\right), A u_{n_{k}}-s_{n_{k}}-A u_{n_{k}}\right\rangle \geq 0, \quad \forall z \in Q .
\end{gathered}
$$

Since $G$ is upper semicontinuous in the first argument and taking lim sup of the above inequality as $k \rightarrow \infty$, we get

$$
G\left(A x^{*}, z\right)+\left\langle z-A x^{*}, g A x^{*}\right\rangle+\varphi(z)-\varphi\left(A x^{*}\right) \geq 0 \quad \forall z \in Q
$$

This implies that $A x^{*} \in \operatorname{GMEP}(G, g, \phi)$. Therefore $x^{*} \in \Omega$, and we can conclude that $x^{*} \in \Psi$. It follows from the existence of $\lim _{n \rightarrow \infty}\left\|x_{n}-x^{*}\right\|$ and the Opial condition that $\left\{x_{n}\right\}$ has only one weak cluster point and hence $\left\{x_{n}\right\}$ converges weakly to $x^{*} \in \Psi$.

The following consequence is a weak convergence theorem for computing a common solution of a split mixed equilibrium problem (for short $\mathrm{S}_{p} \mathrm{MEP}$ ) and a hierarchical fixed point problem in Hilbert spaces.

If we set $\phi=0$ and $\varphi=0$ in (6)-(7), then $\mathrm{S}_{p}$ GMEP is reduced to the split mixed equilibrium problem (in short $\mathrm{S}_{p} \mathrm{MEP}$ ): Find $x^{*} \in C$ such that

$$
F\left(x^{*}, u\right)+\left\langle f x^{*}, u-x^{*}\right\rangle \geq 0 \quad \forall u \in C,
$$

and such that

$$
A x^{*} \in Q \text { solves } \quad G\left(A x^{*}, v\right)+\left\langle g\left(A x^{*}\right), v-A x^{*}\right\rangle \geq 0, \quad \forall v \in Q .
$$

The solution set of the $S_{p}$ MEP is denoted by

$$
\Omega_{1}=\left\{x^{*} \in \operatorname{Sol}(\operatorname{MEP}(42)): A x^{*} \in \operatorname{Sol}(\operatorname{MEP}(43))\right\} .
$$

Corollary 3.2 Let $C$ and $Q$ be nonempty closed and convex subsets of real Hilbert spaces $H_{1}$ and $H_{2}$, respectively, and let $A: H_{1} \rightarrow H_{2}$ be a bounded linear operator with its adjoint operator $A^{*}$. Assume that $F: C \times C \rightarrow \mathbb{R}$ and $G: Q \times Q \rightarrow \mathbb{R}$ are bifunctions satisfying Assumption 3.1. Let $f: C \rightarrow H_{1}$ and $g: Q \rightarrow H_{2}$ be $\kappa_{1}, \kappa_{2}$-inverse strongly monotone mappings, and let $S: C \rightarrow C$ be a nonexpansive mapping and $\left\{T^{i}\right\}_{i=1}^{N}: C \rightarrow H_{1}$ be $k_{i}$-strictly 
pseudocontractive nonself-mappings. Assume that $\Psi_{1}=\Omega_{1} \cap \Phi \neq \emptyset$. Let $\left\{x_{n}\right\}$ be the sequence defined by

$$
\left\{\begin{array}{l}
w_{n}=x_{n}+\theta_{n}\left(x_{n}-x_{n-1}\right) \\
u_{n}=\left(1-\alpha_{n}\right) w_{n}+\alpha_{n}\left(\beta_{n} S w_{n}+\left(1-\beta_{n}\right) T_{n}^{N} \cdots T_{n}^{1} w_{n}\right) ; \\
x_{n+1}=U\left(u_{n}+\delta_{n} A^{*}(V-I) A u_{n}\right), \quad n \geq 1,
\end{array}\right.
$$

where $U=S_{r_{n}}^{F}\left(I-r_{n} f\right), V=S_{r_{n}}^{G}\left(I-r_{n} g\right), T_{n}^{i}=\left(1-\gamma_{n}^{i}\right) I+\gamma_{n}^{i} P_{C}\left(\tau_{n}^{i} I+\left(1-\tau_{n}^{i}\right) T^{i}\right), 0 \leq k_{i} \leq \tau_{n}^{i}<1$, $\gamma_{n}^{i} \in(0,1), \delta_{n}=\frac{\sigma_{n}\left\|\left(T_{r_{n}}^{G}\left(I-r_{n} g\right)-I\right) A x_{n}\right\|^{2}}{\left\|A^{*}\left(T_{r_{n}}^{G}\left(I-r_{n} g\right)-I\right) A x_{n}\right\|^{2}}, 0<a \leq \sigma_{n} \leq b<1$, and $\theta_{n}$ satisfies Algorithm 1 , and the following conditions are satisfied:

(i) $\liminf _{n \rightarrow \infty} \alpha_{n}>0, \sum_{n=1}^{\infty} \beta_{n}<+\infty$;

(ii) $\lim _{n \rightarrow \infty} \frac{\left\|u_{n}-w_{n}\right\|}{\alpha_{n} \beta_{n}}=0$;

(iii) $\liminf _{n \rightarrow \infty} r_{n}>0$.

Then the sequence $\left\{x_{n}\right\}$ converges weakly to $x^{*} \in \Psi_{1}$.

\section{Nash-Cournot oligopolistic market equilibrium problem}

In this section, we apply generalized mixed equilibrium problems with Nash-Cournot oligopolistic market equilibrium problems which have been introduced by Cournot and studied by some authors $[19,20,34]$. An oligopolistic market model considers $n$ firms (producers) that produce a common homogeneous commodity. Each firm has a profit function which is the difference between the price and the cost. Each firm attempts to maximize its profit by choosing the corresponding production level on its strategy set.

Consider that there are $n$-firms which produce a common homogenous commodity and the price $p_{i}$ of firm $i$ depends on the total quantity $\sigma=\sum_{i=1}^{n} x_{i}$ of the commodity. Let $h_{i}\left(x_{i}\right)$ denote the cost of the firm $i$ when its production level is $x_{i}$. Suppose that the profit of firm $i$ is given by

$$
f_{i}\left(x_{1}, \ldots, x_{n}\right)=x_{i} p_{i}\left(\sum_{i=1}^{n} x_{i}\right)-h_{i}\left(x_{i}\right), \quad i=1, \ldots, n
$$

where $h_{i}$ is the cost function of firm $i$ that is assumed to be dependent only on its production level.

Let $U_{i} \subset \mathbb{R}$ for $i=1, \ldots, n$ denote the strategy set of the firm $i$. Each firm seeks to maximize its own profit by choosing the corresponding production level under the hypothesis that the production of other firms is parametric input. In this context, a Nash equilibrium is a production pattern in which no firm can increase its profit by changing its controlled variables. Thus under this equilibrium concept, each firm determines its best response given other firms' actions. Mathematically, a point $x^{*}=\left(x_{1}^{*}, x_{2}^{*}, \ldots, x_{n}^{*}\right) \in U=U_{1} \times \cdots \times U_{n}$ is said to be a Nash-equilibrium if

$$
f_{i}\left(x_{1}^{*}, \ldots, x_{i-1}^{*}, y_{i}, \ldots, x_{n}^{*}\right)=f_{i}\left(x_{1}^{*}, \ldots, x_{n}^{*}\right), \quad \forall y_{i} \in U_{i}, \forall i=1, \ldots, n .
$$

When $h_{i}$ is affine, this market problem can be formulated as a special Nash equilibrium problem in $n$-person noncooperative game theory. 
In classical Cournot models [20,34], the price and the cost functions for each firm are assumed to be affine of the forms:

$$
\begin{aligned}
& p_{i}(\sigma)=p(\sigma)=\alpha_{0}-\beta \sigma, \quad \alpha_{0} \geq 0, \beta>0, \text { with } \sigma=\sum_{i=1}^{n} x_{i}, \\
& h_{i}\left(x_{i}\right)=\mu_{i} x_{i}+\xi_{i}, \quad \mu_{i} \geq 0, \xi_{i} \geq 0 \text { for } i=1, \ldots, n .
\end{aligned}
$$

In this case, we take

$$
\begin{aligned}
B & =\left[\begin{array}{ccccc}
2 \beta & 0 & 0 & \ldots & 0 \\
0 & 2 \beta & 0 & \ldots & 0 \\
\ldots & \ldots & \ldots & \ldots & \ldots \\
0 & 0 & 0 & \ldots & 2 \beta
\end{array}\right], \quad \tilde{B}=\left[\begin{array}{ccccc}
0 & \beta & \beta & \ldots & \beta \\
\beta & 0 & \beta & \ldots & \beta \\
\ldots & \ldots & \ldots & \ldots & \ldots \\
\beta & \beta & \beta & \ldots & 0
\end{array}\right], \\
\alpha & =\left(\alpha_{0}, \ldots, \alpha_{0}\right)^{T}, \quad \mu
\end{aligned}
$$

For (45), (46), (47), it has been shown in [20,34], that the problem can be formulated equivalently as the convex quadratic problem

$$
\min _{x \in U}\left\{\frac{1}{2} x^{T} Q x+(\mu-\alpha)^{T} x\right\}
$$

where

$$
Q=\left[\begin{array}{ccccc}
2 \beta & \beta & \beta & \ldots & \beta \\
\beta & 2 \beta & \beta & \ldots & \beta \\
\ldots & \ldots & \ldots & \ldots & \ldots \\
\beta & \beta & \beta & \ldots & 2 \beta
\end{array}\right] .
$$

Since $\beta>0$, we have $Q$ is a symmetric and positive definite matrix. Hence problem (48) has a unique optimal solution which is also the unique equilibrium point of the classical oligopolistic market equilibrium model.

Let

$$
\begin{aligned}
& \alpha^{T}=\left(\alpha_{1}, \alpha_{2}, \ldots,\right), \quad F(x, y)=(-B x+(B+\tilde{B}) y)^{T}(y-x), \\
& f(x)=\tilde{B} x+\alpha, \quad \phi(x)=h(x) .
\end{aligned}
$$

Then the problem of finding a Nash equilibrium point defined by (46) with $F(x, y), f(x)$ given by (45) becomes the following generalized mixed equilibrium:

$$
\left\{\begin{array}{l}
\text { find a point } x^{*} \in U \text { such that } \\
\Phi\left(x^{*}, y\right)=F\left(x^{*}, y\right)+\left\langle f x^{*}, x^{*}-y\right\rangle+\phi(y)-\phi\left(x^{*}\right) \geq 0 \quad \text { for all } y \in C
\end{array}\right.
$$

where $F$ is a bifunction, $f$ is affine, and $\phi$ is a convex function (see [24]). In the literature problem (49) is often called the generalized mixed equilibrium which is more general than the mixed variational inequality (see [20]) because of the appearance of the bifunction $F$. 


\section{Numerical example}

Numerical results are presented in this section to show the efficiency of the proposed method. The MATLAB codes was run in MATLAB version 9.5 (R2018b) on MacBook Pro 13-inch, 2019 with $2.4 \mathrm{GHz}$ Quad-Core Intel Core i5 processor. RAM $8.00 \mathrm{~GB}$. and the stopping criteria $\frac{\left\|x_{n+1}-x_{n}\right\|}{\max \left\{1,\left\|x_{n}\right\|\right\}} \leq 10^{-5}$.

Example 5.1 We apply Theorem 3.1 with Nash-Cournot oligopolistic market equilibrium problem in Sect. 4 when $H_{1}=\mathbb{R}^{N}$ and $H_{2}=\mathbb{R}^{M}$, with the inner product defined by $\langle x, y\rangle=$ $x y, \forall x, y \in \mathbb{R}^{N}$ and the induced usual norm $|\cdot|$, the linear operator $A: \mathbb{R}^{N} \rightarrow \mathbb{R}^{M}$ is given by an $M \times N$ matrix. The bifunction $F$ is given as follows:

$$
F_{i}(x, y)=(-B x+(B+\tilde{B}) y)^{T}(y-x)
$$

where $B$ and $\tilde{B}$ are symmetric positive semidefinite matrices such that $2 B+\tilde{B}$ is a symmetric negative semidefinite matrix. Since $F_{i}(x, y)+F_{i}(y, x)=(y-x)^{T}(2 B+\tilde{B})(y-x)$, then $F_{i}$ is monotone. Similarly, define the bifunction of $G_{j}$ by

$$
G_{j}(u, v)=(D u+\tilde{D} v)^{T}(v-u)
$$

where $\tilde{D}$ is a symmetric positive semidefinite matrix such that $\tilde{D}-D$ is a symmetric negative semidefinite matrix (see [53]).

The feasible sets are

$$
C=\left\{\left(x_{1}, x_{2}, \ldots, x_{N}\right) \in \mathbb{R}^{N}:-5 \leq x_{i} \leq 5, \forall i=1, \ldots, N\right\}
$$

and

$$
Q=\left\{\left(u_{1}, u_{2}, \ldots, u_{M}\right) \in \mathbb{R}^{M}:-2 \leq u_{j} \leq 2, \forall j=1, \ldots, M\right\} .
$$

The mappings $f_{i}: C \rightarrow H_{1}$ and $g_{j}: Q \rightarrow H_{2}$ are defined by $f_{i}(x)=\tilde{B} x+b, g_{j}(u)=\tilde{D} u+d$, $\phi(x)=b x$ for all $x \in C$ and $\varphi(y)=d y$ for all $y \in Q$. Let $S: C \rightarrow C$ be a mapping defined to be $S x=x$, and

$$
T^{n} x= \begin{cases}\frac{1}{n}\left(1, \frac{1}{2}, \frac{1}{3}, \frac{1}{4}, 0,0,0, \ldots\right), & \text { if } x \neq 0 \\ 0, & \text { if } x=0 .\end{cases}
$$

We can see that $T^{n}$ is $\frac{1}{3}$-strictly pseudocontractive with $\bigcap_{n=1}^{\infty} F\left(T^{n}\right)=\{0\}$. Further, we observe that $T^{n}$ is not nonexpansive (see [32]). Set

$$
\beta_{n}=\frac{1}{n^{3}}, \quad \alpha_{n}=\frac{1}{2}, \quad \theta=0.6 \quad \text { for all } n \in \mathbb{N} .
$$

We use the starting point $x_{0}=(2,2, \ldots, 2)^{T} \in \mathbb{R}^{N}$ and $x_{1}=(-2,-2, \ldots,-2)^{T} \in \mathbb{R}^{N}$. The main subproblems were solved with the MATLAB Optimization Toolbox by using the QUADPROG function for the positive semidefinite quadratic function. The entries of matrices $B, \tilde{B}, D, \tilde{D}$ and vectors $b, d$ are generated randomly in the interval $[-1,5]$ respectively. Define matrix $A=\frac{1}{2} G$, where $G_{i, j}=0$ when $i \neq j$. The numerical and graphical results of our algorithm are shown in Figures 1-4 and Table 1. 


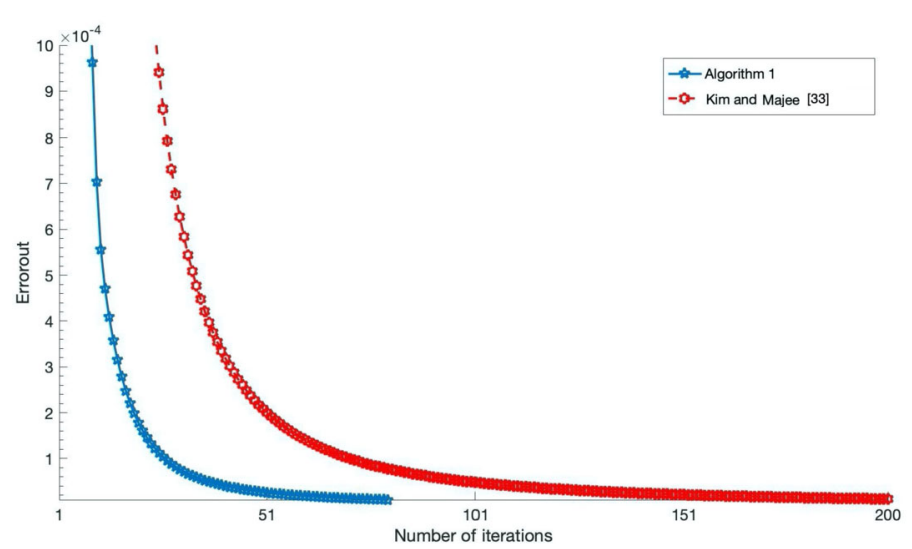

Figure 1 The graph of $N=5, M=5$

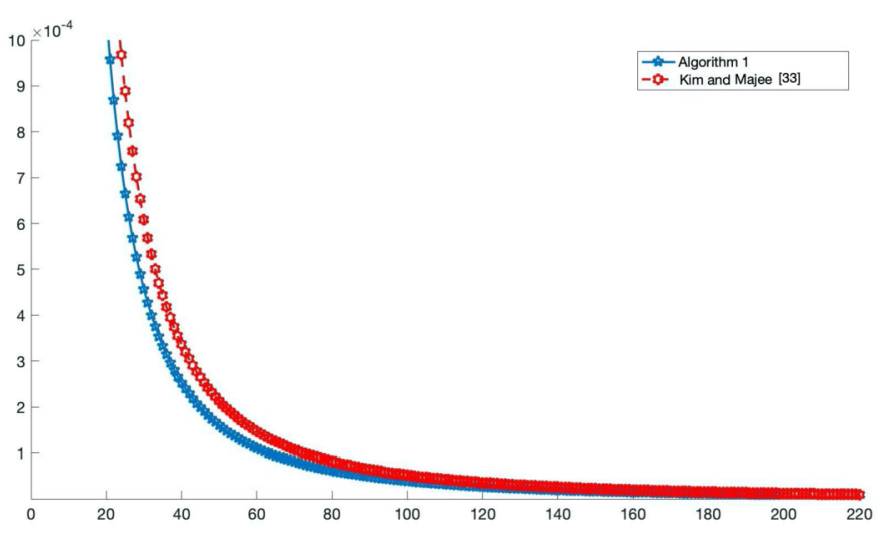

Figure 2 The graph of $N=5, M=10$

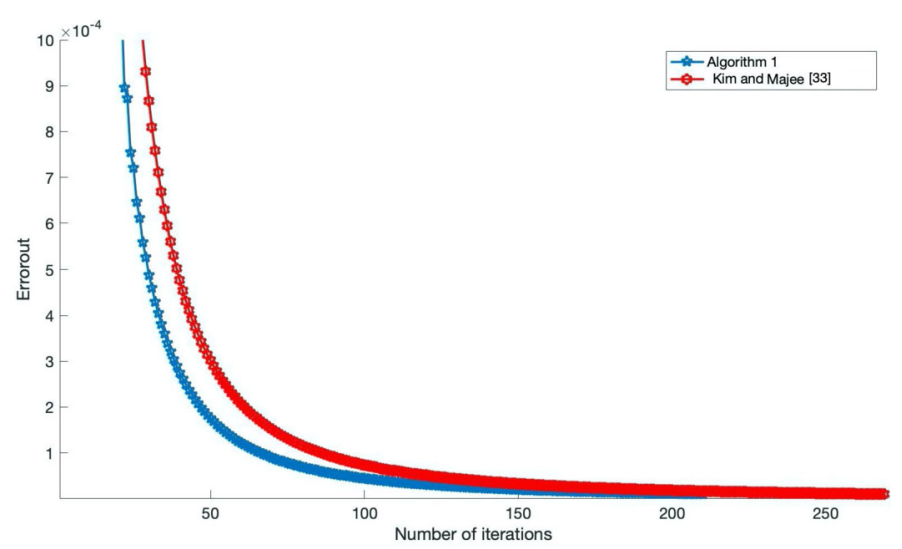

Figure 3 The graph of $N=10, M=20$

Example 5.2 We consider an example in infinite dimensional Hilbert spaces. Assume $H_{1}=$ $H_{2}=L^{2}([0,1])$ with the inner product $\langle x, y\rangle=\int_{0}^{1} x(t) y(t) d t$ and the induced norm $\|x\|=$ 


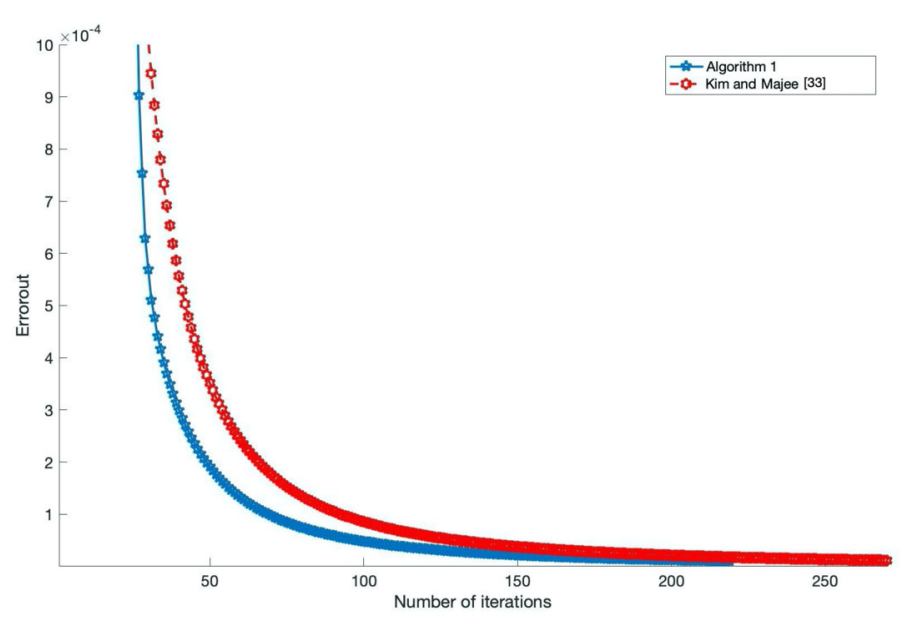

Figure 4 The graph of $N=50, M=100$

Table 1 The results computed on Algorithm 1 and the method in [33]

\begin{tabular}{|c|c|c|c|c|c|}
\hline \multirow[t]{2}{*}{ N } & \multirow[t]{2}{*}{ M } & \multicolumn{2}{|c|}{ Average iteration } & \multicolumn{2}{|c|}{ Average times } \\
\hline & & Algorithm 1 & Method in [33] & Algorithm 1 & Method in [33] \\
\hline 5 & 5 & 77 & 215 & 1.4100 & 3.7300 \\
\hline 5 & 10 & 197 & 227 & 4.0800 & 4.2300 \\
\hline 10 & 20 & 209 & 269 & 4.1000 & 4.3900 \\
\hline 50 & 100 & 218 & 290 & 8.1200 & 10.6600 \\
\hline
\end{tabular}

$\sqrt{\int_{0}^{1}|x(t)|^{2} d t}$ for all $x, y \in L^{2}([0,1])$. Let

$$
C=\left\{x \in L^{2}([0,1]) \mid \int_{0}^{1} x(t) d t \leq 1\right\} \quad \text { and } \quad Q=\left\{u \in L^{2}([0,1]) \mid \int_{0}^{1} t u(t) d t \leq 2\right\}
$$

Therefore $C$ and $Q$ are nonempty closed and convex subsets of real Hilbert spaces $H_{1}$ and $H_{2}$, respectively. Define an operator $A: L^{2}([0,1]) \rightarrow L^{2}([0,1])$ by $(A x)(t)=\frac{1}{2} x(t)$. Thus $A$ is a bounded linear operator. We define $F: C \times C \rightarrow \mathbb{R}$ by

$$
F(x, y)=\langle P(x), y-x\rangle, \quad \text { where } P(x(t))=\frac{x(t)}{2} .
$$

For the purpose of our numerical computation, we use the following formula for the projection onto $C$ (see [5]):

$$
P_{C}(x)= \begin{cases}1-a+x, & \text { if } a>1 \\ x & \text { if } a \leq 1\end{cases}
$$

where $a=\int_{0}^{1} x(t) d t$. Similarly, define the bifunction $G: Q \times Q \rightarrow \mathbb{R}$ by

$$
G(u, v)=\langle H(u), v-u\rangle, \quad \text { where } H(u(t))=\frac{u(t)}{3} .
$$

It is observed that $F$ and $G$ are monotone satisfying conditions L1-L4. Let $S: C \rightarrow C$ be a mapping defined by $(S x)(t)=x(t)$ and $\left(T^{n} x\right)(t)=\frac{3}{n+3} x(t)$. We can see that $T^{n}$ is 0 - 


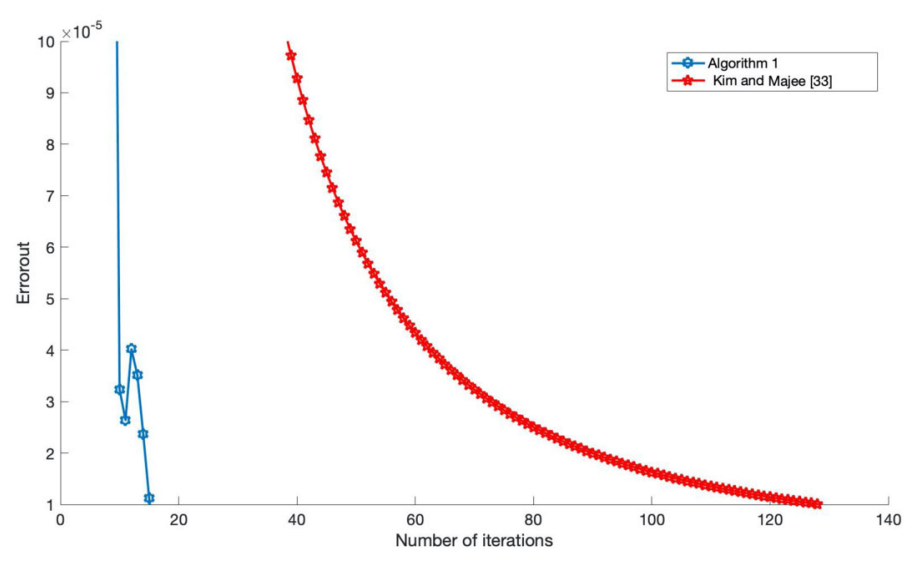

Figure 5 Numerical behaviour when $x_{0}=t, x_{1}=2 t$

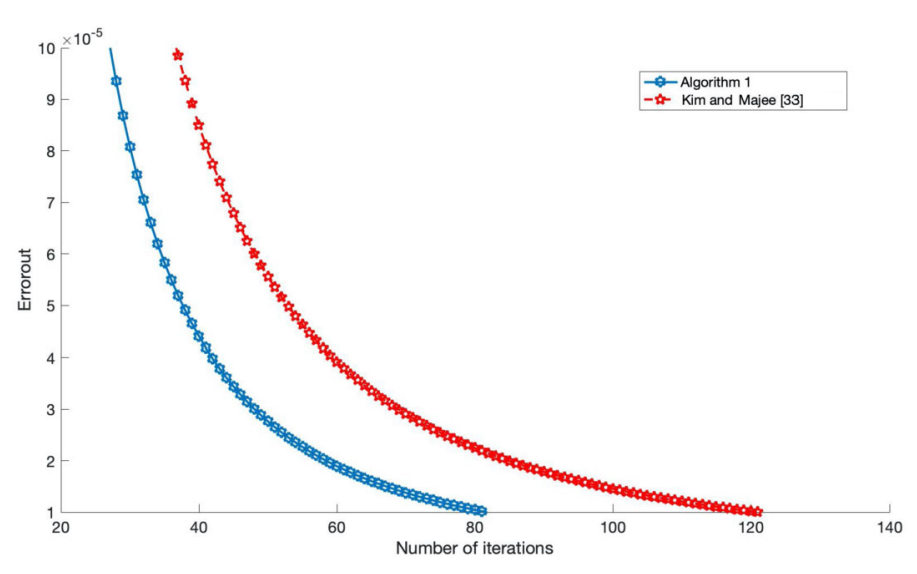

Figure 6 Numerical behaviour when $x_{0}=t, x_{1}=2 t^{2}$

strictly pseudocontractive for all $n \geq 1$. Set $\beta_{n}=\frac{1}{n^{3}}, \alpha_{n}=\frac{1}{2}, \sigma_{n}=0.8, \theta=0.9$ for all $n \in \mathbb{N}$. The mappings $f: C \rightarrow H_{1}$ and $g: Q \rightarrow H_{2}$ are defined by $(f x)(t)=\frac{1}{2} x(t),(g u)(s)=\frac{1}{n} u(s)$, $(\phi(x))(t)=0$ for all $x(t) \in C$ and $(\varphi(u))(t)=0$ for all $u(t) \in Q$. Numerical results are reported in Figs. 5 and 6.

\section{Conclusion}

This paper discussed the modified Krasnoselski-Mann-type iterative method based on the idea of inertial technique. Weak convergence results have been obtained under some suitable conditions. Numerical conclusions have been drawn to explain the numerical efficiency of our algorithm in comparison to another method. Note that our algorithm and results presented in this paper can summarize and improve some known results in the area. Our future work will focus on obtaining the results to robust equilibrium problems by our algorithm in [38, 46, 47].

\section{Acknowledgements}

The authors would like to thank the editor and the anonymous referees for their valuable comments and suggestions which helped to improve the original version of this paper. This work was financially supported by the Faculty of Science, 
Burapha University (Grant no.SC-NO1/2563), and the second author would like to express her deep thanks to the Faculty of Science, Naresuan University (Grant no. R2564E049).

\section{Funding}

This work was supported by the Faculty of Science, Burapha University (Grant no.SC-NO1/2563) and the Faculty of Science, Naresuan University (Grant no. R2564E049).

\section{Availability of data and materials}

Not applicable.

\section{Competing interests}

The authors declare that they have no competing interests.

Authors' contributions

Both authors read and approved the final version of the manuscript.

\section{Author details}

'Department of Mathematics, Faculty of Science, Burapha University, Chonburi, 20131, Thailand. '2Department of Mathematics Faculty of Science, Naresuan University, Phitsanulok, 65000, Thailand.

\section{Publisher's Note}

Springer Nature remains neutral with regard to jurisdictional claims in published maps and institutional affiliations.

\section{Received: 19 January 2021 Accepted: 21 May 2021 Published online: 31 May 2021}

\section{References}

1. Abass, H.A., Jolaoso, L.O.: An inertial generalized viscosity approximation method for solving multiple-sets split feasibility problems and common fixed point of strictly pseudo-nonspreading mappings. Axioms (2021). https://doi.org/10.3390/axioms10010001

2. Alakoya, T.O., Mewomo, O.T: Strong convergence theorems for finite families of pseudomonotone equilibrium and fixed point problems in Banach spaces. Afr. Math. (2021). https://doi.org/10.1007/s13370-020-00869

3. Alakoya, T.O., Taiwo, A., Mewomo, O.T., Cho, Y.J.: An iterative algorithm for solving variational inequality, generalized mixed equilibrium, convex minimization and zeros problems for a class of nonexpansive-type mappings. Ann. Univ. Ferrara, Sez. 7: Sci. Mat. (2020). https://doi.org/10.1007/s11565-020-00354-2

4. Alvarez, F., Attouch, H.: An inertial proximal method for maximal monotone operators via discretization of a nonlinear oscillator with damping. Set-Valued Anal. 9, 3-11 (2001)

5. Bauschke, H.H., Combettes, P.L.: Convex Analysis and Monotone Operator Theory in Hilbert Spaces. Springer, Berlin (2011)

6. Beck, A., Teboulle, M.: A fast iterative shrinkage-thresholding algorithm for linear inverse problems. SIAM J. Imaging Sci. 2, 183-202 (2009)

7. Blum, E., Oettli, W.: From optimization and variational inequalities to equilibrium problems. Math. Stud. $63,123-145$ (1994)

8. Bot, R.I., Csetnek, E.R., Hendrich, C.: Inertial Douglas-Rachford splitting for monotone inclusion problems. Appl. Math. Comput. 256, 472-487 (2015)

9. Brézis, H.: Mathematical Studies 5. Opé rateurs maximaux monotones et semigroupes de contractions dans les espaces de Hilbert. Am. Elsevier, New York (1973)

10. Byrne, C.: A unified treatment of some iterative algorithms in signal processing and image reconstruction. Inverse Probl. 20, 103-120 (2004)

11. Cabot, A.: Proximal point algorithm controlled by a slowly vanishing term: application to hierarchical minimization. SIAM J. Optim. 15, 555-572 (2005)

12. Ceng, L.C., Yao, J.C.: A hybrid iterative scheme for mixed equilibrium problems and fixed point problems. J. Comput. Appl. Math. 214, 186-201 (2008)

13. Censor, Y., Bortfeld, T., Martin, B., Trofimov, A.: A unified approach for inversion problems in intensity-modulated radiation therapy. Phys. Med. Biol. 51(2), 2353-2365 (2006)

14. Censor, Y., Eflving, T:: A multiprojection algorithm using Bregman projections in a product space. Numer. Algorithms $8,221-239(1994)$

15. Censor, Y., Elfving, T., Kopf, N., Bortfeld, T.: The multiple-set split feasibility problem and its application for inverse problems. Inverse Probl. 21(6), 2071-2084 (2005)

16. Censor, Y., Gibali, A., Reich, S.: Algorithms for the split variational inequality problem. Numer. Algorithms 59(2), 301-323 (2012)

17. Censor, Y., Motova, A., Segal, A.: A perturbed projections and subgradient projections for the multiple-set split feasibility problem. J. Math. Anal. Appl. 327, 1244-1256 (2007)

18. Combettes, P.L., Hirstoaga, S.A.: Equilibrium programming in Hilbert spaces. J. Nonlinear Convex Anal. 6(1), 117-136 (2005)

19. Dafermosm, S., Nagurney, A.: Oligopolistic and competitive behavior of spatially separated markets. Reg. Sci. Urban Econ. 17, 225-254 (1997)

20. Facchinei, F., Pang, J.S.: Finite-Dimensional Variational Inequalities and Complementarity Problems. Springer, Berlin (2002)

21. Flam, L.S.D., Antipin, A.S.: Equilibrium programming using proximal like algorithms. Math. Program. 78, 29-41 (1997)

22. Geobel, K., Kirk, W.A.: Topics in Metric Fixed Point Theory. Cambridge University Press, Cambridge (1990) 
23. Giannessi, F.: Theorems of the alternative, quadratic programs and complementarity problems. In: Cottle, R.W., Giannessi, F., Lions, J.L. (eds.) Variational Inequalities and Complementarity Problems, pp. 151-186. Wiley, New York (1980)

24. Horst, R., Tuy, H.: Global Optimization (Deterministic Approach). Springer, Berlin (1990)

25. Jolaoso, L.O.: An inertial projection and contraction method with a line search technique for variational inequality and fixed point problems. Optimization (2021). https://doi.org/10.1080/02331934.2021.1901289

26. Jolaoso, L.O., Alakoya, T.O., Taiwo, A., Mewomo, O.T.: A parallel combination extragradient method with Armijo line searching for finding common solution of finite families of equilibrium and fixed point problems. Rend. Circ. Mat. Palermo 2 69(2), 475-495 (2020)

27. Jolaoso, L.O., Alakoya, T.O., Taiwo, A., Mewomo, O.T.: Inertial extragradient method via viscosity approximation approach for solving equilibrium problem in Hilbert space. Optimization 70(2), 387-412 (2021)

28. Jolaoso, L.O., Aphane, M.: Strong convergence inertial projection and contraction method with self adaptive stepsize for pseudomonotone variational inequalities and fixed point problems. J. Inequal. Appl. 2020, 261 (2020). https://doi.org/10.1186/s13660-020-02536-0

29. Jolaoso, L.O., Aphane, M.: A generalized viscosity inertial projection and contraction method for pseudomonotone variational inequalities and fixed point problems. Mathematics 8(11), 2039 (2020). https://doi.org/10.3390/math8112039

30. Jolaoso, L.O., Oyewole, K.O., Okeke, C.C., Mewomo, O.T:: A unified algorithm for solving split generalized mixed equilibrium problem and for finding fixed point of nonspreading mapping in Hilbert spaces. Demonstr. Math. 51, 211-232 (2018)

31. Kazmi, K.R., Ail, R., Furkan, M.: Krasnoselski-Mann type iterative method for hierarchical fixed point problem and split mixed equilibrium problem. Numer. Algorithms (2017). https://doi.org/10.1007/s11075-017-0316-y

32. Khuangsatung, W., Sunthrayuth, P.: The generalized viscosity explicit rules for a family of strictly pseudo-contractive mappings in a q-uniformly smooth Banach space. J. Inequal. Appl. (2018). https://doi.org/10.1186/s13660-018-1760-5

33. Kim, J.K., Majee, P.: Modified Krasnoselski-Mann iterative method for hierarchical fixed point problem and split mixed equilibrium problem. J. Inequal. Appl. (2020). https://doi.org/10.1186/s13660-020-02493-8

34. Konnov, I.: Combined Relaxation Methods for Variational Inequalities. Springer, Berlin (2001)

35. Lorenzand, D.A., Pock, T.: An inertial forward-backward algorithm for monotone inclusions. J. Math. Imaging Vis. 51(2), 311-325 (2015)

36. Luo, Z.Q., Pang, J.S., Ralph, D.: Mathematical Programs with Equilibrium Constraints. Cambridge University Press, Cambridge (1996)

37. Maingé, P.E.: Convergence theorems for inertial KM-type algorithms. J. Comput. Appl. Math. 219(1), $223-236$ (2008)

38. Minh, N.B., Phuong, T.T.T.: Robust equilibrium in transportation networks. Acta Math. Vietnam. 45, 635-650 (2020)

39. Moudafi, A., Mainge, P.E.: Towards viscosity approximations of hierarchical fixed-point problems. Fixed Point Theory Appl. 2006, Article ID 95453 (2006)

40. Moudafi, A., Oilny, M.: Convergence of a splitting inertial proximal method for monotone operator. J. Comput. Appl. Math. 155, 447-454 (2003)

41. Olona, M.A., Alakoya, T.O., Owolabi, A.O.-E., Mewomo, O.T.: Inertial shrinking projection algorithm with self-adaptive step size for split generalized equilibrium and fixed point problems for a countable family of nonexpansive multivalued mappings. Demonstr. Math. (2021). https://doi.org/10.1515/dema-2021-0006

42. Olona, M.A., Alakoya, T.O., Owolabi, A.O.-E., Mewomo, O.T.: Inertial algorithm for solving equilibrium, variational inclusion and fixed point problems for an infinite family of strictly pseudocontractive mappings. J. Nonlinear Funct. Anal. 2, Article ID 10 (2021)

43. Owolabi, A.O.-E., Alakoya, T.O., Taiwo, A., Mewomo, O.T.: A new inertial-projection algorithm for approximating common solution of variational inequality and fixed point problems of multivalued mappings. Numer. Algebra Control Optim. (2021). https://doi.org/10.3934/naco.2021004

44. Peng, J.W., Yao, J.C.: A new hybrid-extragradient method for generalized mixed equilibrium problems,fixed point problems and variational inequality problems. Taiwan. J. Math. 12(6), 1401-1432 (2008)

45. Sun, X.K., Chai, Y.: Gap functions and error bounds for generalized vector variational inequalities. Optim. Lett. 8, $1663-1673(2014)$

46. Sun, X.K., Teo, K.L., Tang, L.P.: Dual approaches to characterize robust optimal solution sets for a class of uncertain optimization problems. J. Optim. Theory Appl. 182(3), 984-1000 (2019)

47. Sun, X.K., Teo, K.L., Long, X.J.: Characterizations of robust $\varepsilon$-quasi optimal solutions for nonsmooth optimization problems with uncertain data. Optimization 70(4), 847-870 (2021)

48. Taiwo, A., Alakoya, T.O., Mewomo, O.T.: Strong convergence theorem for fixed points of relatively nonexpansive multi-valued mappings and equilibrium problems in Banach spaces. Asian-Eur. J. Math. (2020). https://doi.org/10.1142/S1793557121501370

49. Taiwo, A., Alakoya, T.O., Mewomo, O.T.: Halpern-type iterative process for solving split common fixed point and monotone variational inclusion problem between Banach spaces. Numer. Algorithms (2020) https://doi.org/10.1007/s11075-020-00937-2

50. Taiwo, A., Jolaoso, L.O., Mewomo, O.T., Gibali, A.: On generalized mixed equilibrium problem with $\alpha-\beta-\mu$ bifunction and $\mu-\tau$ monotone mapping. J. Nonlinear Convex Anal. 21(6), 1381-1401 (2020)

51. Taiwo, A., Jolaoso, L.O., Mewomo, T.: Inertial-type algorithm for solving split common fixed point problem in Banach spaces. J. Sci. Comput. 86, Article ID 12 (2020). https://doi.org/10.1007/s10915-020-01385-9

52. Takahashi, L.S., Takahashi, W.: Strong convergence theorem for a generalized equilibrium problem and a nonexpansive mapping in a Hilbert space. Nonlinear Anal. 69, 1025-1033 (2008). https://doi.org/10.1016/j.na.2008.02.042

53. Tran, D.Q., Pham, N.A., Le, D.M.: Dual extragradient algorithms extended to equilibrium problems. J. Glob. Optim. 52, 139-159 (2012)

54. Yamada, I., Ogura, N.: Hybrid steepest descent method for the variational inequality problem over the fixed point set of certain quasi-nonexpansive mappings. Numer. Funct. Anal. Optim. 25, 619-655 (2004)

55. Yang, Q., Zhao, J.: Generalized KM theorem and their applications. Inverse Probl. 22, 833-844 (2006)

56. Zhou, $\mathrm{H}_{\text {.: }}$ Convergence theorems of fixed points for $\mathrm{k}$-strict pseudo-contractions in Hilbert spaces. Nonlinear Anal. 69(2), 456-462 (2008) 